\title{
Millimeter-wave Radio over Fiber System for Broadband Wireless Communication
}

\author{
Haoshuo Chen, Rujian Lin and Jiajun Ye \\ Shanghai University, Shanghai
}

China

\section{Introduction}

The wireless networking has attracted much interest in past decades, owing to its high mobility. People can connect their devices such as PDAs, mobile phones or computers to a network by radio signals anywhere in home, garden or office without the need for wires. The global growth of mobile subscribers is much faster than wireline ones, as the Figure 1 shows (Yungsoo et al., 2003). The number of mobile subscribers worldwide has increased from 215 million in 1997 to 946 million (15.5\% of global population) in 2001. It is predicted that by the year 2010 there will be 1,700 million terrestrial mobile subscribers worldwide. At present, main wireless standards are Wireless LAN (WLAN), IEEE802.11a/b/g, offering up to 54-Mbps and operating at 2.4-GHz and 5-GHz, and 3-G mobile networks, IMT2000/UMTS, offering up to 2-Mbps and operating around 2-GHz. But with the development of human society, people have higher requirements for the services, such as video, multimedia and other new value-added services. In order to offer these broadband services, wireless systems will need to offer higher data transmission capacities.

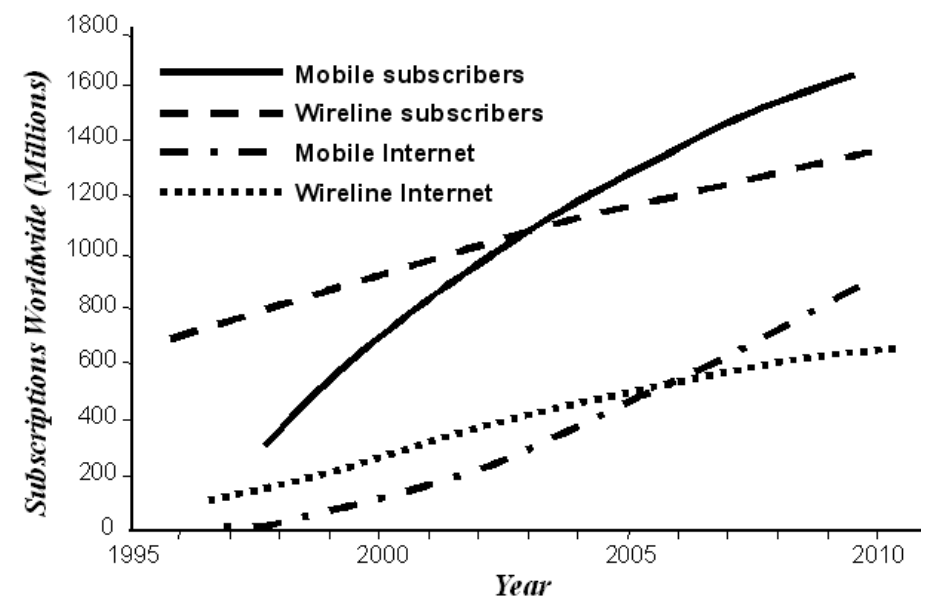

Fig. 1. Global growth of mobile and wireline subscribers. 
By increasing operating frequencies of wireless system, a broader bandwidth can be provided to transmit data with higher transmission speed. In Millimeter-wave (mm-wave) band (30-GHz $\sim 300 \mathrm{GHz}$ ), about $270-\mathrm{GHz}$ bandwidth can be utilized, which is ten times the bandwidth in Centimeter-wave band (3-GHz 30-GHz). Moreover, the increase of operation frequency helps to minimize the size of wireless equipment and improve the antenna directivity. But free space loss increases drastically with frequency and obstacles such as a human body may easily cause a substantial drop of received power at mm-wave band, nullifying the gain provided by the antennas. Besides, the diffraction of mm-wave, the ability to bend around edges of obstacles is weak (Smulders, 2002). Due to the characteristics of mm-wave, the electrical delivery of mm-wave wireless signals over a long distance is not feasible. Many research works have been done to transmit mm-wave over the fiber-optic links, which exploit the advantages of both optical fibers and $\mathrm{mm}$-wave frequencies to realize broadband communication systems and contribute a lot to the development of mmwave Radio over Fiber (RoF) systems (Sun et al., 1996; Braun et al., 1998; Kitayama, 1998).

Figure 2 gives the architecture of mm-wave RoF system. Central Station (CS) and distributed Base Stations (BS) are linked with optical fibers. In each pico-cell, BS communicates with some Mobile Terminals (MT) by wireless signals at mm-wave band.

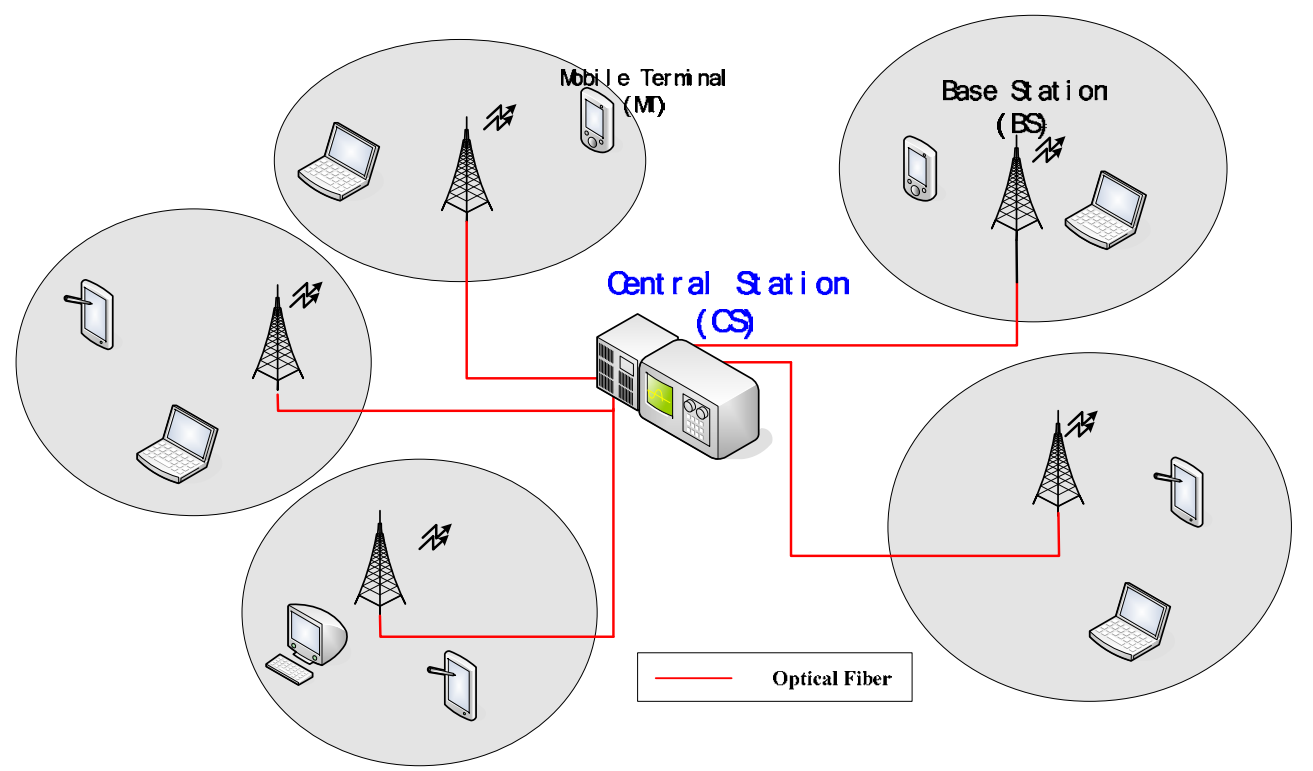

Fig. 2. Architecture of mm-wave RoF system.

Main issues in mm-wave RoF system include the optical methods of generating low noise mm-wave wireless signal and overcoming the influence of fiber chromatic dispersion on the transmission of optical wireless signal. Because of the great amounts of BSs, to reduce the system's capital, installation and maintenance costs, it is imperative to make the distributed BSs as simple as possible. Therefore, the signal processing works, such as modulation/demodulation for information conveying, cross-cell handover control, and etc. should be centralized on CS, making the BS be a simple light-wave to mm-wave converter. 
In this chapter, a brief introduction of mm-wave RoF system will be given and the optical techniques of generating mm-wave signals are presented. Unlike the conventional discussions about mm-wave RoF systems focusing on the downlink only, the design of bidirectional mm-wave RoF systems are considered. Two multiplexing techniques, Wavelength Division Multiplexing (WDM) and Subcarrier Multiplexing (SCM) are introduced to realize the distributed BSs. Fiber chromatic dispersion, the main cause of performance degradation in optical communications also affects mm-wave RoF systems, making the mm-wave fade with distance in the fiber links. The influence of fiber chromatic dispersion on different mm-wave generation techniques will be discussed. The Medium Access Control (MAC) protocols suitable for the fast handover of mm-wave systems are also introduced.

\section{Techniques of millimeter-wave signal generation in RoF Systems}

The generation of mm-wave wireless signal in BS using optical techniques is the key technical issue of mm-wave RoF systems. In the following context, three optical technologies to yield mm-wave signal, such as direct intensity modulation, optical self-heterodyning and Optical Frequency Multiplication (OFM) will be introduced.

\subsection{Direct intensity modulation and external intensity modulation}

The direct intensity modulation is realized by applying mm-wave directly to the laser and change the intensity of the launched light, the mm-wave signal can be recovered in BS by direct detection. Hartmannor et al. (2003) reported the experimental reuslt of using uncooled directly modualted DFB lasers to transmit high data-rate Orthogonal Frequency Division Multiplexing (OFDM) video signals over 1-km multi-mode fiber (MMF). The experimental setup is shown in Figure 3. The video signal is transmitted from a mobile laptop to a desktop PC.

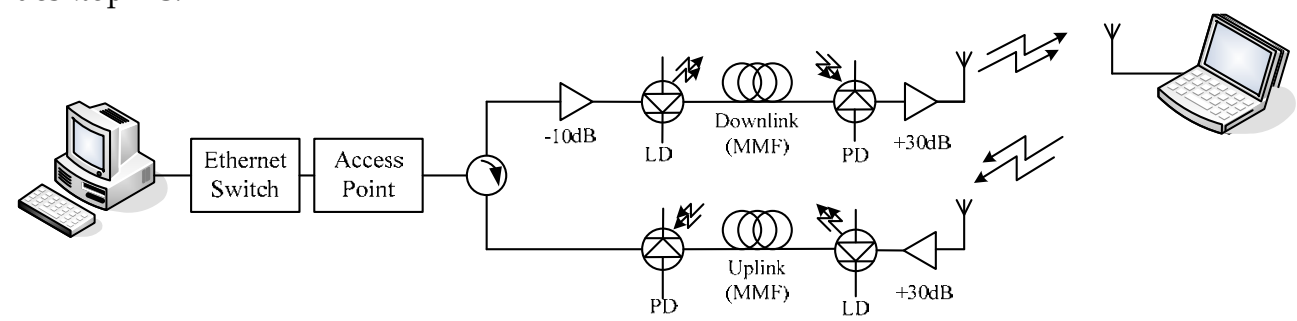

Fig. 3. The experimental setup of direct intensity modulation.

The main drawback of direct intensity modulation is that the bandwidth of modulating signal is limited by the modulation bandwidth of laser.

Another way to realize intensity modulation is to modulate the light launched from a laser which operates in continuous wave (CW) mode in an external intensity modulator, e.g., Mach-Zehnder modulator (MZM) or electro-absorption modulator (EAM). Figure 4 gives the scheme of generating mm-wave signal by using MZM (O'Rcilly et al., 1992). 


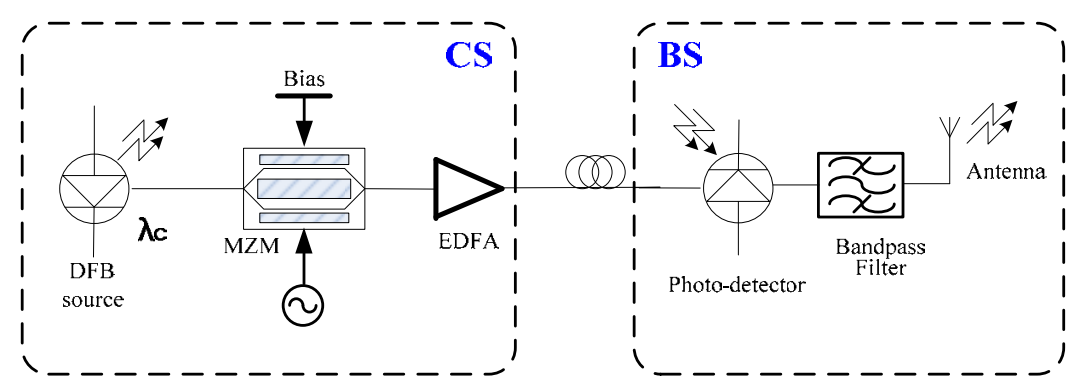

Fig. 4. The usage of $\mathrm{MZM}$ as external modulator.

It can be seen that a single laser source is required together with a MZM. By biasing the MZM at Vpi, the half-wave voltage of MZM, the optical carrier at center wavelength will be suppressed. The beat of upper and lower 1st side-modes will yield required mm-wave signal, whose frequency is twice that of the mm-wave signal applied to MZM. The EAM is also a candidate of external modulator (Kuri et al., 1999). The mm-wave produced by these intensity modulation schemes have some advantages such as no line-width broadening due to the fiber dispersion, but a mm-wave oscillator is required in CS inevitably, which is costly.

\subsection{Optical self-heterodyning}

The generation of mm-wave signal by self-heterodyning of two-mode light-waves has a good effect to overcome the fiber chromatic dispersion (Gliese et al., 1996).

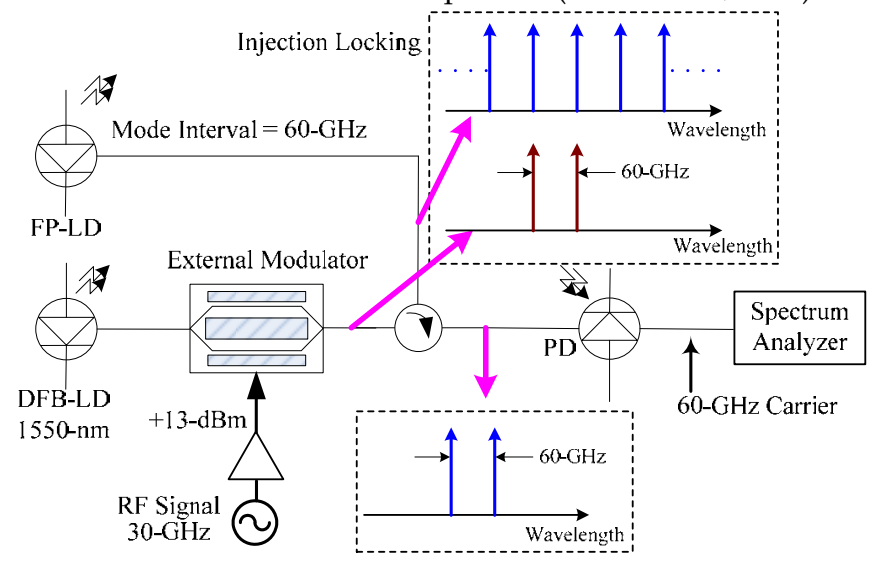

Fig. 5. Configuration of the two-mode injection-locking of a FP LD for optical selfheterodyning

Optical self-heterodyning is based on transmission of two phase-correlated optical signals, at frequencies $f_{1}$ and $f_{2}$. The difference of these two frequencies is the frequency of desired $\mathrm{mm}$-wave signal. After opto-electronic conversion at photodiode (PD) in BS, the mm-wave at frequency $\mathrm{f}_{\mathrm{c}}\left(\mathrm{f}_{\mathrm{c}}=\mathrm{f}_{1}-\mathrm{f}_{2}\right)$ is generated. Figure 5 shows the configuration of the two-mode locking of a FP LD to generate $60-\mathrm{GHz} \mathrm{mm}$-wave carrier. The 1st upper and lower sidemodes, obtained by applying MZM with $30-\mathrm{GHz}$ radio frequency (RF) signal to modulate 
the CW output from a DFB LD are used to lock the two modes of the FP LD, whose mode interval is $60-\mathrm{GHz}$ (Ogusu et al., 2003). The main drawback of optical self-heterodyning is the strong influence of laser phase noise and optical frequency variation on the purity and stability of the generated mm-wave signal. The optical phase-locked loop (OPLL) has been used to reduce the phase noise (Williams et al., 1989; Gliese et al., 1992). Hence the optical self-heterodyning is a costly solution for photonic generation of mm-wave signal because it needs a special laser system.

\subsection{Optical Frequency Multiplication (OFM)}

\subsubsection{OFM by optical frequency sweeping technique}

Optical Frequency Multiplication (OFM) is a kind of photonic methods which up-convert low frequency microwave into mm-wave band.The mm-wave generation by OFM is based on a technique called as optical frequency sweeping which is ideally implemented by launching light-wave from a fast tunable laser which is swept periodically at a microwave frequency, but this technique is infeasible, because this kind of tunable laser is unavailable in the market.

Figure 6 gives an arrangement of OFM by an alternate optical frequency sweeping technique. It can be seen that in CS a light-wave is launched by a laser diode operating at CW mode and then phase-modulated in an extennal phase modulator by a microwave signal at frequency $f_{\mathcal{S}}$ with a large modulation index. The output light-wave becomes an optical frequency-swept signal, having a lot of side modes separated by $f s$. This phase spectrum is converted into an intensity spectrum by passing the phase-modulated lightwave through a periodic optical filter such as Mach-Zehnder interferometer (MZI). These intensity side modes beat with each other at the PD in BS producing a series of harmonics of the sweeping signal. In this way, a mm-wave at frequency $2 n f s$ is generated which can be picked up using a narrowband band-pass filter and amplified for radiating into the air via an antenna. In this scheme, the data signal can be intensity-modualted on the optical frequency-swept signal by an external intensity modulator (Ton et al., 2003). The principle of OFM is deduced as below.

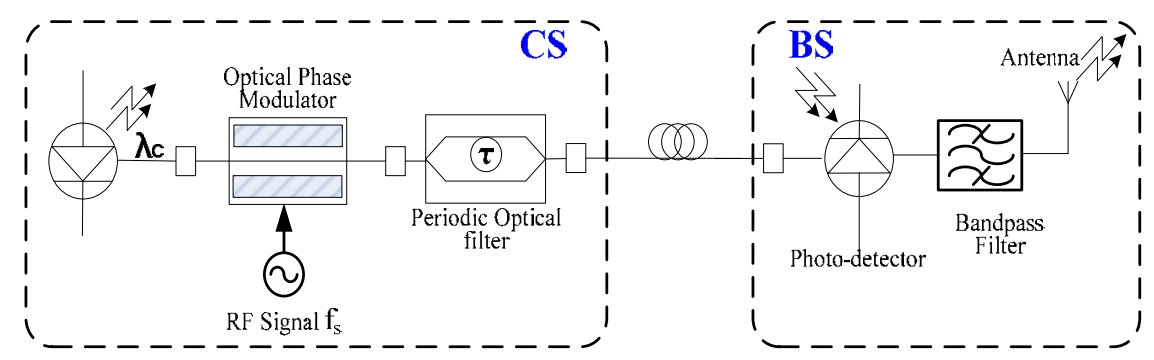

Fig. 6. The basic arrangement of OFM using optical frequency sweeping technique.

The electric field at the output of optical phase modulator is express as

$$
E_{i}(t)=E_{c} \exp \left(j \omega_{c} t+j \beta \sin \omega_{s} t\right)
$$


where $E_{c}$ is the amplitude of electric field; $\omega_{c}$ is the central angular frequency of optical source; $\omega_{s}$ is the angular frequency of phase sweeping signal; $\beta$ is the phase modulation index. After passing through the MZI with delay parameter $\tau$, the electric field becomes

$$
E_{o}(t)=E_{c} \exp \left(j \omega_{c} t+j \beta \sin \omega_{s} t\right)+E_{c} \exp \left[j \omega_{c}(t-\tau)+j \beta \sin \omega_{s}(t-\tau)\right]
$$

The photo-current is proportional to $E_{o}(t) \times E_{o}^{*}(t)$, i.e.

$$
\begin{aligned}
i_{d}(t)= & \frac{1}{2} R\left\{E_{o}(t) \times E_{o}^{*}(t)\right\}=\frac{1}{2} R E_{c}^{2}(t)\left\{1+\cos \left[\omega_{c} \tau+2 \beta \sin \left(\frac{\omega_{s} \tau}{2}\right) \cos \left(\omega_{s} t-\frac{\omega_{s} \tau}{2}\right)\right]\right\} \\
= & \frac{1}{2} R E_{c}^{2}(t)\left\{\cos \left(\omega_{c} \tau\right)\left[\mathrm{J}_{0}\left(2 \beta \sin \frac{\omega_{s} \tau}{2}\right)+2 \sum_{n=1}^{\infty} \mathrm{J}_{2 n}\left(2 \beta \sin \frac{\omega_{s} \tau}{2}\right) \cos \left(2 n \omega_{s} t-n \omega_{s} \tau\right)\right]\right. \\
& \left.-\sin \left(\omega_{c} \tau\right)\left[2 \sum_{n=1}^{\infty} \mathrm{J}_{2 n-1}\left(2 \beta \sin \frac{\omega_{s} \tau}{2}\right) \cos \left((2 n-1) \omega_{s} t-\frac{2 n-1}{2} \omega_{s} \tau\right)\right]\right\}
\end{aligned}
$$

where $R$ is a proportional constant related to the responsivity of PD. $\mathrm{J}_{\mathrm{n}}(\mathrm{x})$ is the $\mathrm{n}$-th Bessel function of $\mathrm{x}$. From (3) it is revealed that if $\omega_{c} \tau=k \pi,\left(k=\right.$ integer) and $\omega_{s} \tau=\pi$, each of even harmonics in the photo-current approaches its maximum value while all odd harmonics disappear, i.e.

$$
i_{d}(t)=\frac{1}{2} R E_{c}^{2}\left[\mathrm{~J}_{0}(2 \beta)+2 \sum_{n=1}^{\infty}(-1)^{n} \mathrm{~J}_{2 n}(2 \beta) \cos \left(2 n \omega_{s} t\right)\right]
$$

This means that the central wavelength $\lambda_{c}$ of laser source and the delay constant $\tau$ of MZI should be kept to meet a specific relation, otherwise the mm-wave generation will not be effective. For example, the parameters of system in Figure 6 are taken as: $f_{\mathrm{s}}=5 \mathrm{GHz}, \tau=0.1$ ns, $\lambda_{c}=2 c \tau / k$, where $c$ is the light velocity in vacuum. If $k=38706,38707,38708$, Then $\lambda_{c}=1550.147 \mathrm{~nm}, 1550.107 \mathrm{~nm}, 1550.067 \mathrm{~nm}$ respectively. This means that $\lambda_{c}$ deviates from its optimum value by $0.02 \mathrm{~nm}$ will cause the desired harmonic to disappear.

Although this technique does not need any mm-wave oscillator and up-conversion chain both in CS and in BS, the trouble in the real situation exists such as the temperature dependence of $\tau$. If one wants the system stable, the MZI should be temperature stabilized in addition to that the laser should be wavelength-tunable. Hence this kind of OFM configuration for mm-wave generation is not cost-effective (Lin et al., 2008).

\subsubsection{OFM by nonlinear modulation of dual-drive Mach-Zehnder modulator}

Recognizing that there are two basic processes for OFM: one, optical phase modulation with large modulation index to generate high order optical side-modes; another, phase modulation-to-intensity modulation conversion to make self-heterodyne happen at PD. For the implementation of phase modulation-to-intensity modulation conversion it is necessary to have two laser beams interfering with each other, but MZI optical filter is not the only device to make optical interference.

Actually a dual-drive Mach-Zehnder modulator (DD-MZM) is a parallel combination of two optical phase modulators and its two arms can make optical interference happen. Therefore 
an OFM arrangement to generate mm-wave based on high order optical side-modes generation \& self-heterodyne using a DD-MZM can be configured as Figure 7.

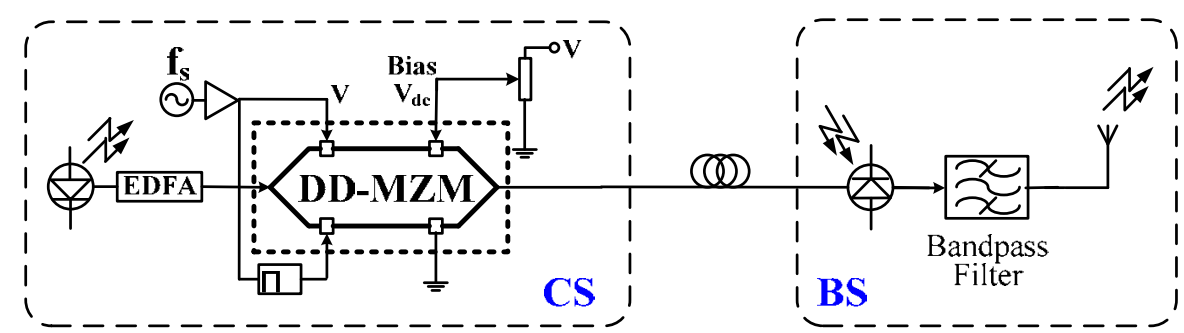

Fig. 7. The basic arrangement of OFM by nonlinear modulation of DD-MZM.

DD-MZM as a commonly used linear intensity modulator can also output optical wave with many harmonics when it is modulated in a nonlinear way, i.e. driven by two large RF signals applied to its two electrodes. Assuming $E_{c}$, the amplitude of electric field input to two arms of DD-MZM; $\tau$, the time delay difference between two arms of DD-MZM; $\theta$, the phase difference between two RF signals; $\Delta \varphi_{d c}$, the initial phase difference of light-waves in the two arms of DD-MZM; $\beta$, the phase modulation index caused by the RF signals; $\omega_{c}$ and $\omega_{s}$, the angular frequencies of the light-wave and the RF signal respectively; $\varphi_{\mathrm{N}}(t+\tau)$ and $\varphi_{\mathrm{N}}(t)$, the laser phase noise in two arms of DD-MZM, the electrical field of output light-wave from DD-MZM is

$$
\begin{aligned}
E_{o}= & E_{c} \exp \left[j \omega_{c}(t+\tau)+j \beta \cos \left(\omega_{s}(t+\tau)+\theta\right)+\Delta \varphi_{d c}+\varphi_{\mathrm{N}}(t+\tau)\right] \\
& +E_{c} \exp \left[j \omega_{c} t+j \beta \cos \omega_{s} t+\varphi_{\mathrm{N}}(t)\right]
\end{aligned}
$$

The photo-current $i_{d}(t)$ in PD produced by light-wave injection is

$$
\begin{aligned}
& i_{d}(t)=\frac{1}{2} R E_{o} E_{o}^{*}=R E_{c}{ }^{2}\left\{1+\cos \left[\beta \cos \left(\omega_{s} t+\omega_{s} \tau+\theta\right)\right.\right. \\
&\left.\left.-\beta \cos \omega_{s} t+\omega_{c} \tau+\Delta \varphi_{d c}+\varphi_{\mathrm{N}}(t+\tau)-\varphi_{\mathrm{N}}(t)\right]\right\}
\end{aligned}
$$

Setting $\Delta \varphi=\omega_{c} \tau+\Delta \varphi_{d c}, \alpha=\omega_{s} \tau+\theta, i_{d}(t)$ can be simplified into the form following:

$$
\begin{aligned}
i_{d}(t)=R E_{c}{ }^{2}\left\{1+\cos \left[\beta_{1} \cos \left(\omega_{s} t+\alpha\right)-\beta_{2} \cos \omega_{s} t+\Delta \varphi+\varphi_{\mathrm{N}}(t+\tau)-\varphi_{\mathrm{N}}(t)\right]\right\} \\
=R E_{c}{ }^{2}+R E_{c}{ }^{2}\left\{\cos \left[\Delta \varphi+\varphi_{\mathrm{N}}(t+\tau)-\varphi_{\mathrm{N}}(t)\right] \cos \left[\beta_{12} \cos \left(\omega_{s} t+\phi\right)\right]\right. \\
\left.-\sin \left[\Delta \varphi+\varphi_{\mathrm{N}}(t+\tau)-\varphi_{\mathrm{N}}(t)\right] \sin \left[\beta_{12} \cos \left(\omega_{s} t+\phi\right)\right]\right\}
\end{aligned}
$$

where $\beta_{12}=\beta\left[(\cos \alpha-1)^{2}+(\sin \alpha)^{2}\right]^{1 / 2}, \phi=\operatorname{tg}^{-1}[-\sin \alpha /(\cos \alpha-1)]$. In a DD-MZM, the two arms are identical in length, therefore $\tau=0$, the laser phase noise terms in photo-current is 
cancelled with each other. In addition, if $\alpha=\omega_{s} \tau+\theta=\pi$ holds, then $\beta_{12}$ reaches its maximum value $2 \beta$ and $\phi=0$.

Expanding $i_{d}(t)$ into a series by using Bessel function gives

$$
\begin{aligned}
i_{d}(t)=R E_{c}^{2}+R E_{c}^{2}\left\{\cos \Delta \varphi\left[J_{0}(2 \beta)+2 \sum_{n=1}^{\infty}(-1)^{n} J_{2 n}(2 \beta) \cos \left(2 n \omega_{s} t\right)\right]\right. \\
\left.\left.+\sin \Delta \varphi\left[2 \sum_{n=1}^{\infty}(-1)^{n} J_{2 n-1}(2 \beta) \cos \left((2 n-1) \omega_{s} t\right)\right]\right\}\right\}
\end{aligned}
$$

The photo-current is composed of a lot of even order harmonics and odd order harmonics of the RF signal. By adjusting the bias voltage $V_{d c}$ to make that $\Delta \varphi=\omega_{c} \tau+\Delta \varphi_{d c}=\pi V_{d c} / V_{\pi}=0$ or $\pi, \cos \Delta \varphi= \pm 1$ and $\sin \Delta \varphi=0$, the odd order harmonics disappear, while each even order harmonic reaches its maximum, i.e.

$$
i_{d}(t)=R E_{c}^{2}\left[1 \pm J_{0}(2 \beta) \pm 2 \sum_{n=1}^{\infty}(-1)^{n} J_{2 n}(2 \beta) \cos \left(2 n \omega_{s} t\right)\right]
$$

where $\beta=\pi V / V_{\pi}, V$ is the amplitude of RF signal, $V_{\pi}$ is the voltage for $\pi$ phase shift of MZM. If a specific value of $(2 \beta)$ is taken, then the specific order Bessel function reaches its maximum. For example, to generate $40-\mathrm{GHz}$ carrier from $5-\mathrm{GHz}$ signal (multiplying factor $2 n=8)$, setting $\beta=4.8$ gives $J_{8}(9.6)=0.3244$. The maximum amplitude of generated $40-\mathrm{GHz}$ carrier is $0.6488 \times R E_{c}^{2}$.

In comparison with the OFM scheme shown in Figure 6, OFM by nonlinear modulation of DD-MZM has the following advantages:

(1) The optimum condition to make odd harmonics disappear and even harmonics maximum is independent of the laser wavelength so that the tunable laser is no longer necessary.

(2) The system does not need any periodic optical filter, such as MZI, to implement PM-IM conversion, so that the system stability is improved by getting rid of temperature-sensitive devices. Even the phase shift in each arm of DD-MZM also depends on the environmental temperature, the two arms with the same length are integrated together in a compact package so that the influences of temperature variation on the two phase modulators are in balance and will be cancelled out.

(3) Laser phase noise is cancelled out so that the output spectrum of mm-wave is pure.

(4) The configuration of CS is most simplified by excluding the tunable DFB LD and the MZI, so that cost saving is achieved.

Because the harmonics are generated in the condition that the phase modulation index is high, a high power amplifier is required to amplify the RF signal. Many works have been done to lower the driving voltage of MZM. A push-pull structure MZM with $V_{\pi}$ of $0.3 \mathrm{~V}$ has been proposed by Tsuzuki et al. (2006). 


\section{Bi-directional mm-wave RoF System based on OFM by using DD-MZM}

\subsection{Bi-directional mm-wave RoF system based on OFM with IF sub-carrier}

In addition to the photonic generation of mm-wave, an important thing is how to convey baseband information on a mm-wave. One technique is to directly modulating the mmwave by the baseband information, another is to modulate the mm-wave by an intermediate frequency (IF) sub-carrier which conveys the base-band information. The advantages of utilizing an IF signal consist in that first, various modulation/demodulation schemes for conveying the base-band information can be used as required by applications; second, the IF signal inserted in the system can also be a pilot tone to generate a pure mm-wave in BS which will be used as a local reference signal for down-converting the modulated mm-wave received from the antenna back into an modulated IF signal; third, utilization of a group of IF sub-carriers instead of one can more sufficiently exploit the bandwidth of mm-wave.

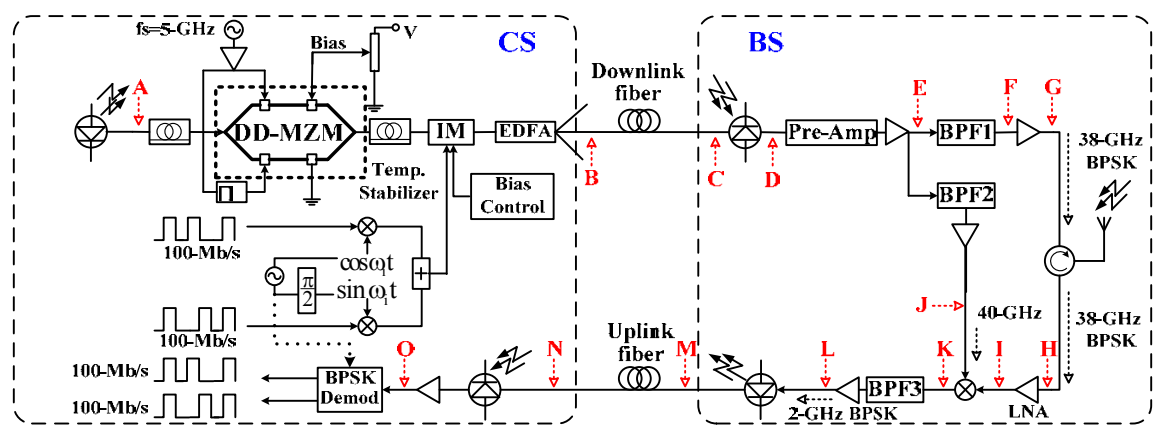

Fig. 8. Bidirectional 38/40-GHz RoF system based on OFM using DD-ZM

Considering the cost-efficiency of bi-directional mm-wave RoF systems, the BS should be configured as simple as possible and no complex electronic circuits, such as mm-wave local oscillator, mm-wave up-conversion chain is needed in both CS and BS. Therefore, the modulated mm-wave signal for radiation in downlink and the mm-wave reference for downconverting the received mm-wave signal into IF signal in uplink need to be generated at the same time after opto-electronic conversion in BS. Then the down-converted signal can be easily modulated on the light-wave launched by a low cost FP laser and sent back to CS. A bidirectional 40-GHz RoF system based on high order optical side-modes generation \& selfheterodyne using a DD-MZM can be configured as Figure 8 with observation points A, B, C... O. In downlink, a 1550-nm polarization-adjusted laser beam in CS is injected into a DD-MZM, whose two arms are DC-biased properly and RF-driven separately. Two 5-GHz sinusoidal waves with phase difference $\pi$ are driving the DD-MZM to carry out optical phase modulation with a large index in each arm. At the combining point of DD-MZM, the two optical beams with different phases interfere with each other converting optical phase modulation into optical intensity modulation with many high order side-modes. These optical modes are re-modulated in another IM by an information-bearing 2-GHz IF signal and then transmitted over a downlink fiber of $20-\mathrm{km}$. Finally they beat at the PD in BS, generating many electrical harmonics of $5-\mathrm{GHz}$ signal, among which any harmonic can be picked up by a specific narrowband band-pass filter. In this way not only a pure $40-\mathrm{GHz}$ signal, but also a 38$\mathrm{GHz}$ mm-wave carrying $2 \times 100-\mathrm{Mbps}$ Ethernet data in BPSK format is generated. The latter 
will be amplified and radiated into the air via an antenna. In uplink, the 38-GHz signal from the antenna is amplified by a low noise amplifier (LNA) and then mixed with the amplified local 40-GHz signal, resulting in a 2-GHz IF signal. The filtered and amplified IF signal directly modulates a FP LD, being sent back to CS via the uplink fiber and recovered at PD. Eventually the amplified IF signal is BPSK-demodulated into $2 \times 100-$ Mbps Ethernet data. The modulating IF signal which carries the digital information in phase is expressed as

$$
m(t)=\cos \left(\omega_{i} t+\phi_{1}\right)+\sin \left(\omega_{i} t+\phi_{\mathrm{Q}}\right)
$$

where $\omega_{i}$ is the angular frequency of IF signal; $\phi_{\mathrm{I}}$ and $\phi_{\mathrm{Q}}$ are the in-phase and quadrature symbols of information carried by the IF signal. Including the modulation effect in IM, the expression of photo-current given in (9) is modified as

$$
\begin{aligned}
& i(t)=R E_{c}{ }^{2}[1+k m(t)]\left[1 \pm \mathrm{J}_{0}(2 \beta) \pm 2 \sum_{n=1}^{\infty} \mathrm{J}_{2 n}(2 \beta) \cos \left(2 n \omega_{s} t\right)\right] \\
& \pm k\left\{\sum_{n=1}^{\infty} \mathrm{J}_{2 n}(2 \beta) \cos \left[\left(2 n \omega_{s}+\omega_{i}\right) t+\phi_{\mathrm{I}}\right]+\sum_{n=1}^{\infty} \mathrm{J}_{2 n}(2 \beta) \cos \left[\left(2 n \omega_{s}-\omega_{i}\right) t-\phi_{\mathrm{I}}\right]\right\} \\
& \pm k\left\{\sum_{n=1}^{\infty} \mathrm{J}_{2 n}(2 \beta) \sin \left[\left(2 n \omega_{s}+\omega_{i}\right) t+\phi_{\mathrm{Q}}\right]+\sum_{n=1}^{\infty} \mathrm{J}_{2 n}(2 \beta) \sin \left[\left(2 n \omega_{s}-\omega_{i}\right) t-\phi_{\mathrm{Q}}\right]\right\}
\end{aligned}
$$

where $k$ is the intensity modulation index in IM. Obviously the photo-current contains not only the IF side-bands centered at each harmonic of the RF signal, but also the pure harmonics of the RF signal. The latter can be used as the local reference signal for downconversion process in reception from the antenna.

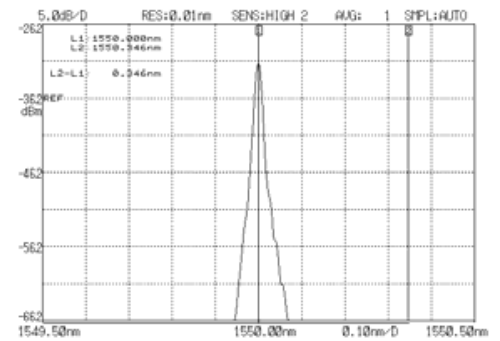

(a)

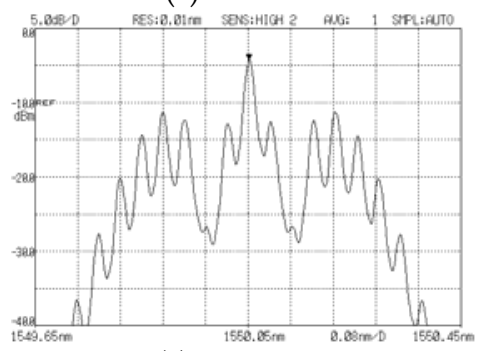

(c)

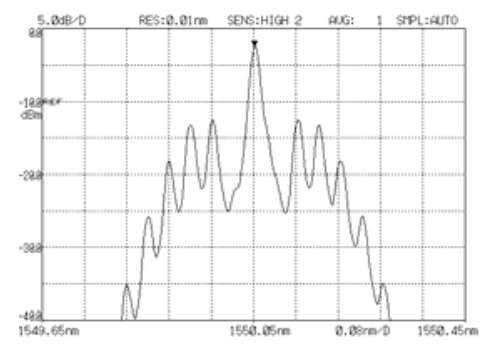

(b)

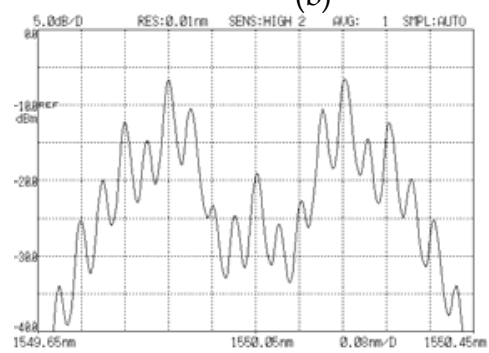

(d)

Fig. 9. The optical spectrum of (a) laser source at $A,(b)$ at $C$ when single arm of DD-MZM driven by $+24 \mathrm{dBm}$, (c) at $C$ when single arm driven by $+27 \mathrm{dBm},(\mathrm{d})$ at $C$ when dual arms driven by $+27 \mathrm{dBm}$. 
The experimental results are shown below. Figure 9 (a) shows the optical spectrum of the DFB laser. Figures 9 (b) and (c) show the optical spectrum expansion as the optical phase modulation index increases in case that one arm of DD-MZM is driven. When the $5-\mathrm{GHz}$ driving power approaches to $+27 \mathrm{dBm}$, the \pm 4 th side-modes rise to the highest indicating $\beta=4.8$, as shown in Figure 9 (c). Applying this best driving power to both arms of DD-MZM, the optical spectrum shown in Figure 9 (d) becomes a carrier suppressed type with strong side-modes around \pm 20 -GHz.

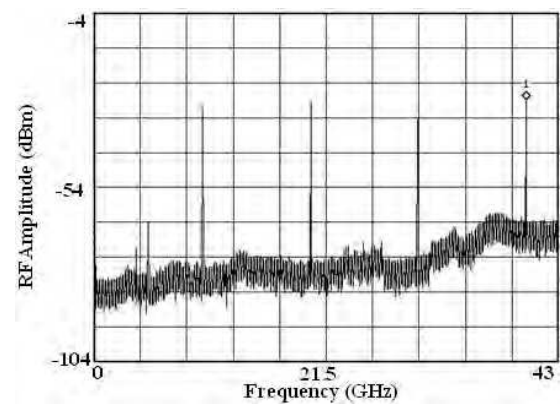

(a)

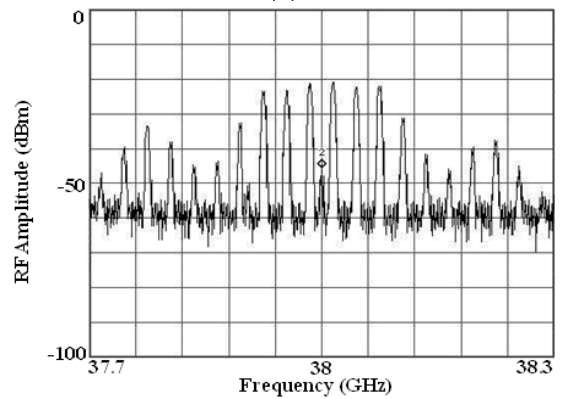

(c)

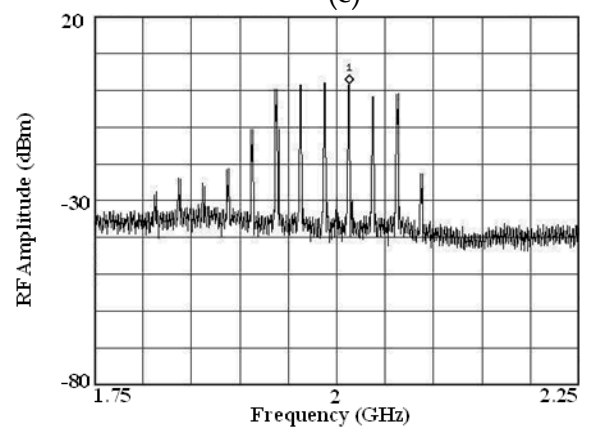

(e)

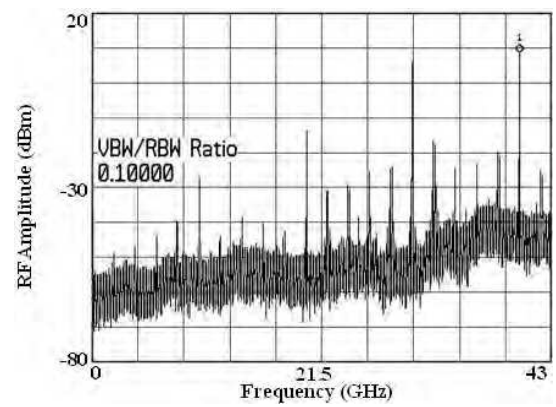

(b)

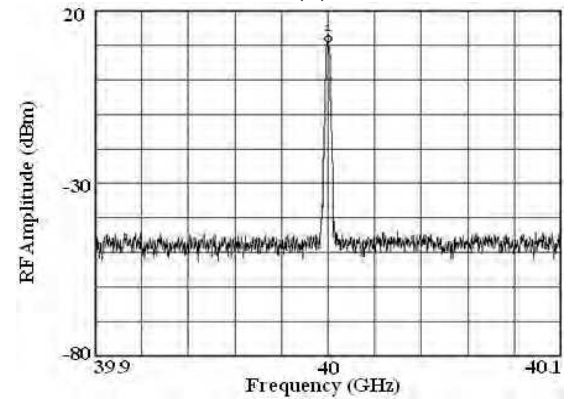

(d)

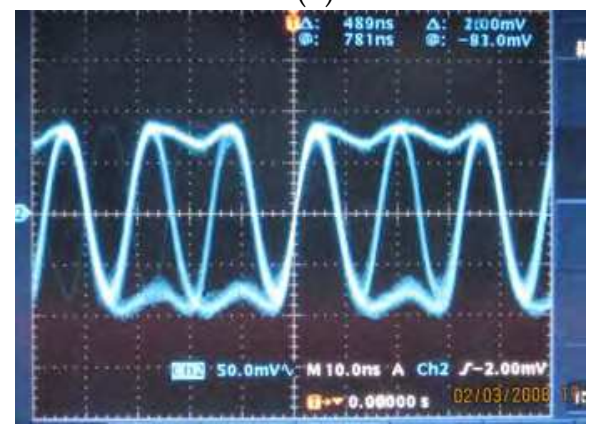

(f)

Fig. 10. The electrical spectrum of (a) generated harmonics at D, (b) at D when 2-GHz IF signal is added, (c) 38-GHz BPSK at E, (d) 40-GHz signal at J, (e) 2-GHz BPSK at O, (f) Waveform of demodulated 100-Mbps Ethernet data. 
It is expected that strong $40-\mathrm{GHz}$ mm-wave will be generated at PD in BS. This is proved by the RF spectrum at PD output, as shown in Figure 10 (a) where $40-\mathrm{GHz}$ peak is the highest among other harmonics and odd harmonics disappear, because the bias voltage has been adjusted to meet $\Delta \varphi=\pi$. To maintain this optimum operating condition the DD-MZM has been put in a temperature stabilizer. When the BPSK-modulated 2-GHz IF signal is turned on, its spectrum appears around each generated harmonic, as shown in Figure 10 (b). Figure 10 (c) shows the spectrum of 38-GHz BPSK signal at point $\mathrm{E}$ in Figure 8. The filtered $40-\mathrm{GHz}$ signal is amplified to above $+10-\mathrm{dBm}$ at point $\mathrm{J}$ with carrier-to-noise ratio (CNR) larger than $50-\mathrm{dB}$ as shown in Figure 10 (d), and is good as a local signal for the mixer. The 2-GHz BPSK signal from the mixer is amplified, transmitted over the uplink fiber and recovered in CS, as shown in Figure 10 (e). Figure 10 (f) shows the BPSK-demodulated 100-Mbps Ethernet data, giving the evidence that the proposed bidirectional $40-\mathrm{GHz}$ RoF system is successful.

In this kind of bidirectional mm-wave RoF systems, the base-band digital information is conveyed by IF signal, on which many modulation/de-modulation schemes, such as PSK, QPSK, M-QAM and even OFDM can be used. In other words, this kind of bidirectional mmwave RoF systems is transparent to the base-band information and very flexible to various applications. Larrode et al. (2006) demonstrated the generation of a 39.9-GHz mm-wave based on OFM with 1.5-GHz IF sub-carrier, which is 16 or 64 QAM-modulated.

\subsection{Bi-directional mm-wave RoF system with QPSK direct modulation}

Although bidirectional mm-wave RoF systems based on OFM with IF sub-carrier are advantageous in many respects, the width of an IF band limits very high speed transmission over the RoF system. Quadrature phase-shift keying (QPSK) is much more widely used than BPSK, since QPSK modulation scheme encodes two bits per symbol, which is twice the rate of BPSK. The QPSK-modulated IF signal has been employed in mm-wave RoF system. Now optical QPSK can be used to generate QPSK-modulated mm-wave signal. A system has been realized by using two optical fiber links to transmit I and Q signals separately (Fuster et al., 2001), but two PDs in BS have to be utilized, therefore it is not cost-effective.

Figure 11 gives a new design of $60-\mathrm{GHz}$ bi-directional RoF system, whose modulation scheme is optical QPSK, similar to a previous work (Zhou et al., 2008), but two DD-MZMs in parallel connection are replaced by a four electrodes DQPSK Lithium Niobate (LN) modulator (Doi et al., 2007), which can greatly overcome the problem of interference intensity noise (IIN) caused by the time delay between the two optical paths.

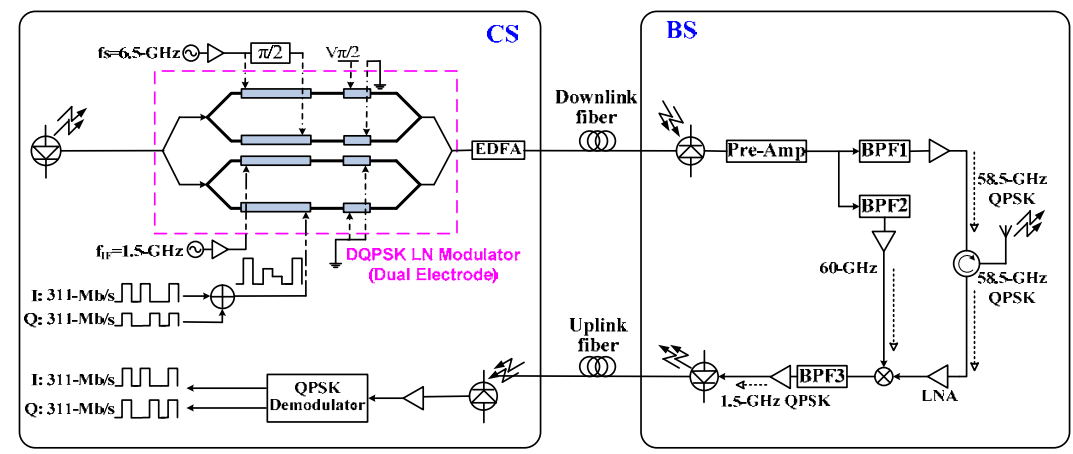

Fig. 11. The basic structure of optical QPSK bidirectional mm-wave RoF system. 
As shown in Figure 11, two high-power 6.5-GHz RF signals with phase difference $\pi / 2$ are applied to one MZI's two electrodes of DQPSK modulator. And a 1.5-GHz IF signal for generating pure mm-wave tone and 622-Mbit/s baseband signal, after 2 to 4 level conversion drive another MZI's two electrodes respectively. After photo-detection at the PD in BS, the 58.5-GHz QPSK-modulated signal and pure $60-\mathrm{GHz}$ signal are generated. This configuration of mm-wave generation is analyzed as below. The output electric field from the DQPSK modulator is expressed as

$$
\begin{aligned}
E_{\text {out }}(t) & =E_{c} \exp \left[j \omega_{c} t+j \beta \cos \omega_{s} t+j \pi / 2+j \varphi_{N}(t)\right] \\
& +E_{c} \exp \left[j \omega_{c} t+j \beta \sin \omega_{s} t+j \varphi_{N}(t)\right] \\
& +E_{c} \exp \left[j \omega_{c} t+j \alpha \cos \omega_{I F} t+\varphi_{N}(t)\right] \\
& +E_{c} \exp \left[j \omega_{c} t+j \phi_{M}+j \varphi_{N}(t)\right]
\end{aligned}
$$

where $E_{c}$ is the amplitude of electric field; $\omega_{c}$ is the angular frequency of light source; $\omega_{s}$ is the the angular frequency of RF signal; $\omega_{I F}$ is the the angular frequency of IF signal; $\beta$ and $\alpha$ are the phase modulation indexes of RF signal and IF signal respectively; $\varphi_{N}(t)$ is the laser phase noise; $\varphi_{M}$ is the QPSK phase symbol with random value taken in $\{0, \pi / 2, \pi$, $3 \pi / 2\}$. The time delay difference between any two arms in the integrated DQPSK modulator can be neglected.

The photo-current in BS is $i_{d}(t)=0.5 \times R E_{\text {out }}(t) \times E_{\text {out }} *(t)$. Substituting (12) for $E_{\text {out }}(t)$ gives $i_{d}(t)$ in the expression as following

$$
\begin{aligned}
i_{d}(t)= & R E_{c}^{2}\left\{2+2 \sum_{n=1}^{\infty} \mathrm{J}_{2 n-1}(\sqrt{2} \beta) \sin \left[(2 n-1)\left(\omega_{s} t-\frac{\pi}{4}\right)\right]+2 \mathrm{~J}_{0}(\alpha) \sum_{n=1}^{\infty}(-1)^{n} \mathrm{~J}_{2 n-1}(\beta) \cos \left[(2 n-1) \omega_{s} t\right]\right. \\
& +2 \mathrm{~J}_{1}(\alpha) \cos \left(\omega_{I F} t\right)\left[\mathrm{J}_{0}(\beta)+2 \sum_{n=1}^{\infty}(-1)^{n} \mathrm{~J}_{2 n}(\beta) \cos \left(2 n \omega_{s} t\right)\right] \\
& +2 \cos \phi_{M} \sum_{n=1}^{\infty}(-1)^{n} \mathrm{~J}_{2 n-1}(\beta) \cos \left[(2 n-1) \omega_{s} t\right]+\sin \phi_{M}\left[\mathrm{~J}_{0}(\beta)+2 \sum_{n=1}^{\infty}(-1)^{n} \mathrm{~J}_{2 n}(\beta) \cos \left(2 n \omega_{s} t\right)\right] \\
& +\mathrm{J}_{0}(\alpha)\left[\mathrm{J}_{0}(\beta)+2 \sum_{n=1}^{\infty} \mathrm{J}_{2 n}(\beta) \cos \left(2 n \omega_{s} t\right)\right]+4 \mathrm{~J}_{1}(\alpha) \cos \left(\omega_{I F} t\right) \sum_{n=1}^{\infty} \mathrm{J}_{2 n-1}(\beta) \sin \left[(2 n-1) \omega_{s} t\right] \\
& +\cos \phi_{M}\left[J_{0}(\beta)+2 \sum_{n=1}^{\infty} J_{2 n}(\beta) \cos \left(2 n \omega_{s} t\right)\right]+2 \sin \phi_{M} \sum_{n=1}^{\infty} J_{2 n-1}(\beta) \sin \left[(2 n-1) \omega_{s} t\right] \\
& \left.+\cos \phi_{M} J_{0}(\alpha)+2 \sin \phi_{M} J_{1}(\alpha) \cos \left(\omega_{I F} t\right)\right\}
\end{aligned}
$$

where $\cos \left(\alpha \cos \omega_{I F} t\right) \approx \mathrm{J}_{0}(\alpha)$ and $\sin \left(\alpha \cos \omega_{I F} t\right) \approx 2 \mathrm{~J}_{1}(\alpha)$ have been taken, because the function of IF signal in this system is to generate pure mm-wave in BS as local reference signal for reception down-conversion, therefore the modulation index $\alpha$ should be small enough to make the linear modulation of IF signal onto the light-wave.

From (13) it is revealed that only the odd harmonics of RF signal have quadrature components. It means that only the odd harmonics of RF signal can convey the baseband information in QPSK form, whose $(2 \mathrm{n}-1)^{\text {th }}$ components are expressed as 


$$
\begin{aligned}
F_{2 n-1}= & 2 R E_{c}^{2}\left\{\mathrm{~J}_{2 n-1}(\sqrt{2} \beta) \sin \left[(2 n-1)\left(\omega_{s} t-\frac{\pi}{4}\right)\right]+J_{0}(\alpha)(-1)^{n} \mathrm{~J}_{2 n-1}(\beta) \cos \left[(2 n-1) \omega_{s} t\right]\right. \\
& +\cos \phi_{M}(-1)^{n} \mathrm{~J}_{2 n-1}(\beta) \cos \left[(2 n-1) \omega_{s} t\right]+\sin \phi_{M} \mathrm{~J}_{2 n-1}(\beta) \sin \left[(2 n-1) \omega_{s} t\right] \\
& +\mathrm{J}_{1}(\alpha) \mathrm{J}_{2 n-1}(\beta)\left\{\sin \left[\left((2 n-1) \omega_{s}+\omega_{I F}\right) t\right]+\sin \left[\left((2 n-1) \omega_{s}-\omega_{I F}\right) t\right]\right\}
\end{aligned}
$$

where the first two terms are the (2n-1) th harmonics of RF signal; the second two terms represent the QPSK-modulated $(2 n-1)^{\text {th }}$ harmonics of RF signal; the last two terms represent the IF side-bands centered at the (2n-1) th harmonics of RF signal.

If $n=5$, the $9^{\text {th }}$ harmonics of RF signal are expressed as

$$
\begin{array}{rl}
F_{9}=2 & R E_{c}^{2}\left\{\mathrm{~J}_{9}(\sqrt{2} \beta) \sin \left(9 \omega_{s} t-\frac{\pi}{4}\right)-\mathrm{J}_{0}(\alpha) \mathrm{J}_{9}(\beta) \cos \left(9 \omega_{s} t\right)\right. \\
& -\mathrm{J}_{9}(\beta)\left[\cos \phi_{M} \cos \left(9 \omega_{s} t\right)-\sin \phi_{M} \sin \left(9 \omega_{s} t\right)\right] \\
& \left.+\mathrm{J}_{1}(\alpha) \mathrm{J}_{9}(\beta)\left\{\sin \left[\left(9 \omega_{s}+\omega_{I F}\right) t\right]+\sin \left[\left(9 \omega_{s}-\omega_{I F}\right) t\right]\right\}\right\}
\end{array}
$$

Taking $f_{s}=6.5 \mathrm{GHz}, f_{I F}=1.5 \mathrm{GHz}, f_{9}=58.5 \mathrm{GHz}$, Simulation has been performed using VPI software. Figures 12 (a) and (b) shows the optical spectrum of QPSK-modulated 58.5-GHz signal and 60-GHz reference signal for down-conversion in uplink. The QPSK modulated 1.5-GHz signal resulting from down-conversion is shown in Figure 12 (c). The constellation of demodulated 622-Mbit/s QPSK vector is given in Figure 13.

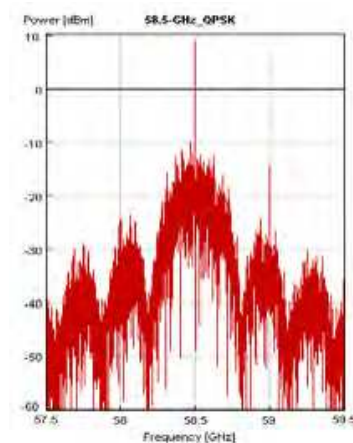

(a)

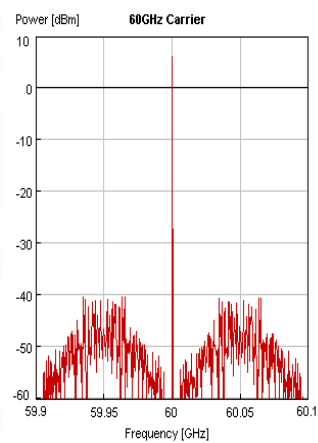

(b)

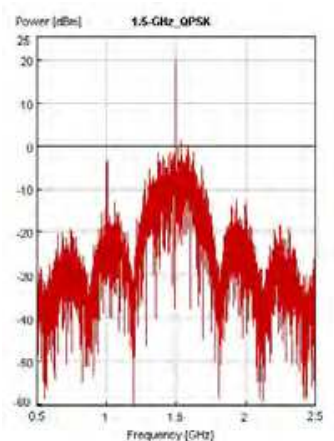

(c)

Fig. 12. The electrical spectrum of (a) QPSK-modulated 58.5-GHz signal, (b) pure 60-GHz carrier, (c) QPSK-modulated 1.5-GHz signal after down-conversion.

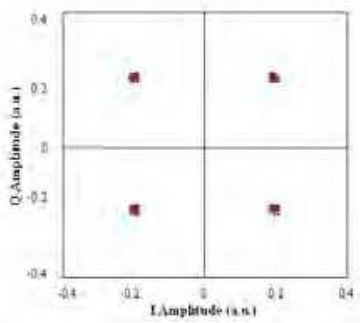

Fig. 13. Constellation of recovered QPSK signal. 


\subsection{Multiplexing of RoF system}

\subsubsection{Subcarrier Multiplexing (SCM) in RoF Link}

SCM technology has been widely used in analog cable television (CATV) (Olshansky et al., 1989). Optical SCM technology which multiplexes various signals in the RF region and transmitted at a single wavelength can also be helpful to improve the bandwidth utilization of mm-wave provided by mm-wave RoF system. Garcia et al. (2005) proposed to apply optical SCM to RoF system based on optical frequcny sweeping technology. In optical frequency sweeping technology, the maximum mm-wave bandwidth supported by one wavelength is limited by half the RF sweep frequncy $f_{s w}$, i.e. if $f_{s w}=5-\mathrm{GHz}$, as described in Section 2.3.1, the maximum mm-wave bandwidth can achieve 2.5-GHz. If the bandwidth of data signals on the sub-carriers exceeds $f_{s w} / 2$, overlapping of the double-sided bands obtained at every harmonic occurs. As shown in Figure 14, data channels on sub-carriers below $f_{s w} / 2$ can be used to modulate the swept light source.

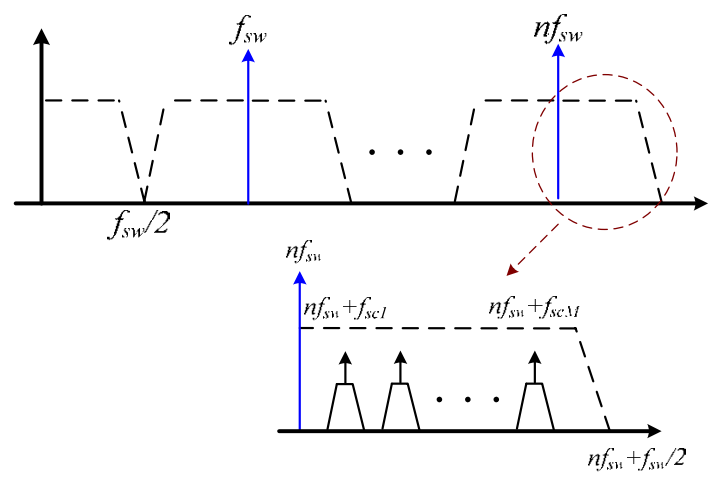

Fig. 14. Bandwidth capacity of optical frequency sweeping technique.

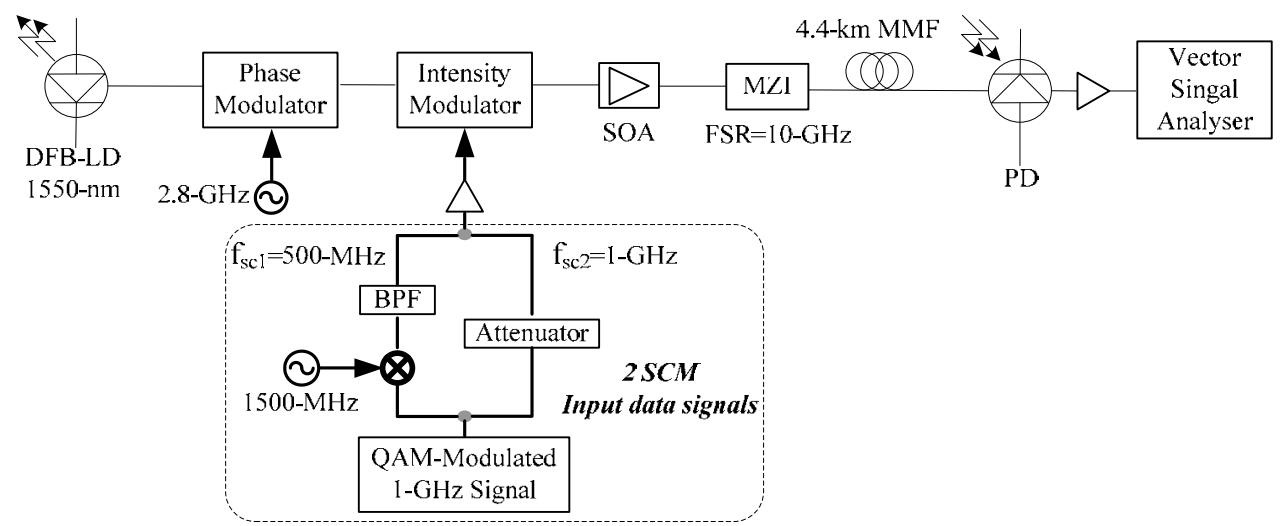

Fig. 15. Experimental setup of SCM RoF system. 
Garcia et al. (2005) set up an experiment to demonstrate the feasibility to transmit the multiple RF signals at a single wavelength. The experimental setup is shown in Figure 15. Two 64 QAM-modulated signals on two sub-carriers at 500-MHz and 1-GHz are transmitted simultaneously in a RoF link. After 4.4-km fiber transmission in a RoF link based optical frequency sweeping, these two 64 QAM-modulated signals can be up-converted to 17.3$\mathrm{GHz}$ and 17.8-GHz.

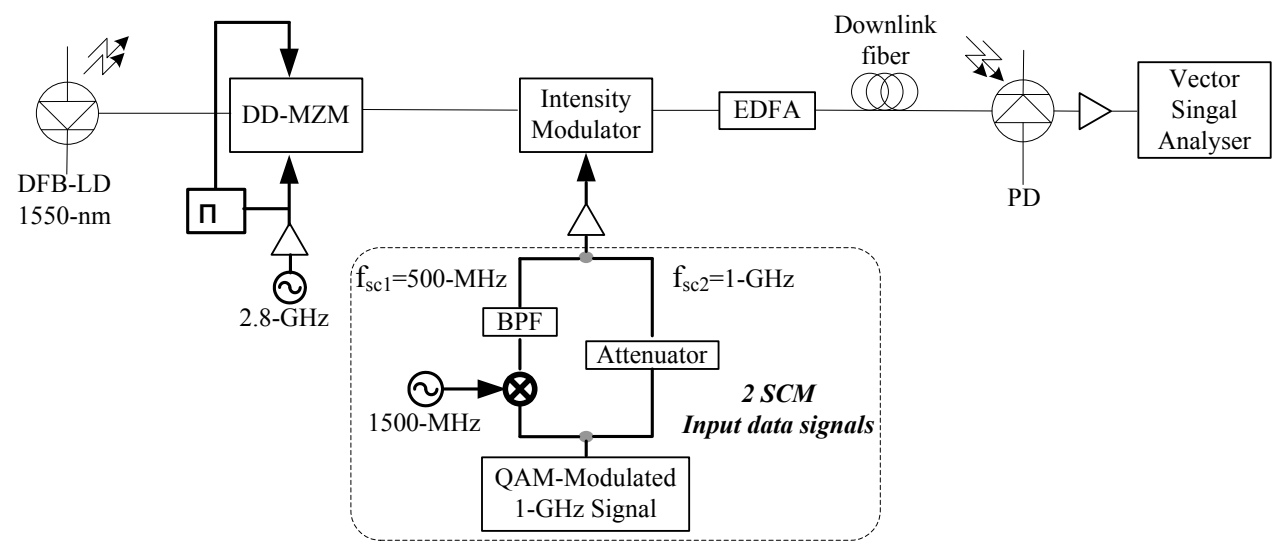

Fig. 16. An alternate setup of SCM RoF system.

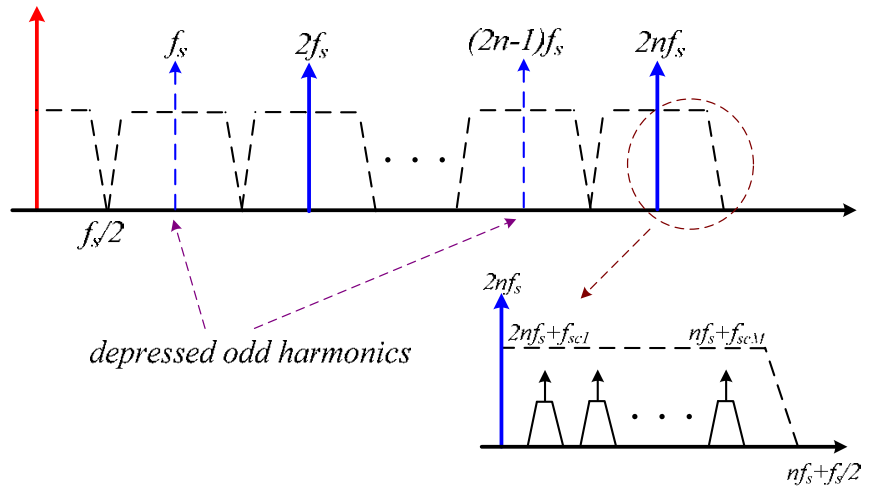

Fig. 17. Bandwidth capacity of OFM by nonlinear modulation of DD-MZM.

In section 2.3.2, it is pointed out that OFM by nonlinear modulation of DD-MZM is much better than OFM by optical frequency sweeping, therefore an alternate setup to Figure 15 is made as Figure 16, where a push-pull driven DD-MZM replaces the phase modulator plus MZI in Figure 15. Although the odd harmonics can be depressed in this scheme, according to equation (9) (in section 2.3.2), the bandwidth capacity of this technique is the same as optical frequency sweeping technique, as shown in Figure 17, $f_{s}$ is the frequency of RF signal applied to DD-MZM. Because the bandwidth capacity is decided by the bandwidth of each optical side-mode, generated by nonlinear modulation of DD-MZM, rather than that of generated mm-wave harmonics after opto-electronic conversion. 


\subsubsection{Wavelength Division Multiplexing (WDM) in RoF Link}

The bidirectional RoF systems, discussed in Section 3.1 and 3.2, have been demonstrated as a cost-effective scheme to generate mm-wave signal and realize bidirectional transmission. All those proposed systems can also realize the architecture with distributed BSs operating at one wavelength. As depicted in Figure 18, CS broadcasts the data packets at one wavelength to all BSs. Each BS extracts its own packets and transmits the data signals, which are up-converted to mm-wave band, sent to MTs. In other words, CS allocates different time slots for different BSs, and makes the RoF system work in Time Division Multiplexing (TDM) scheme.

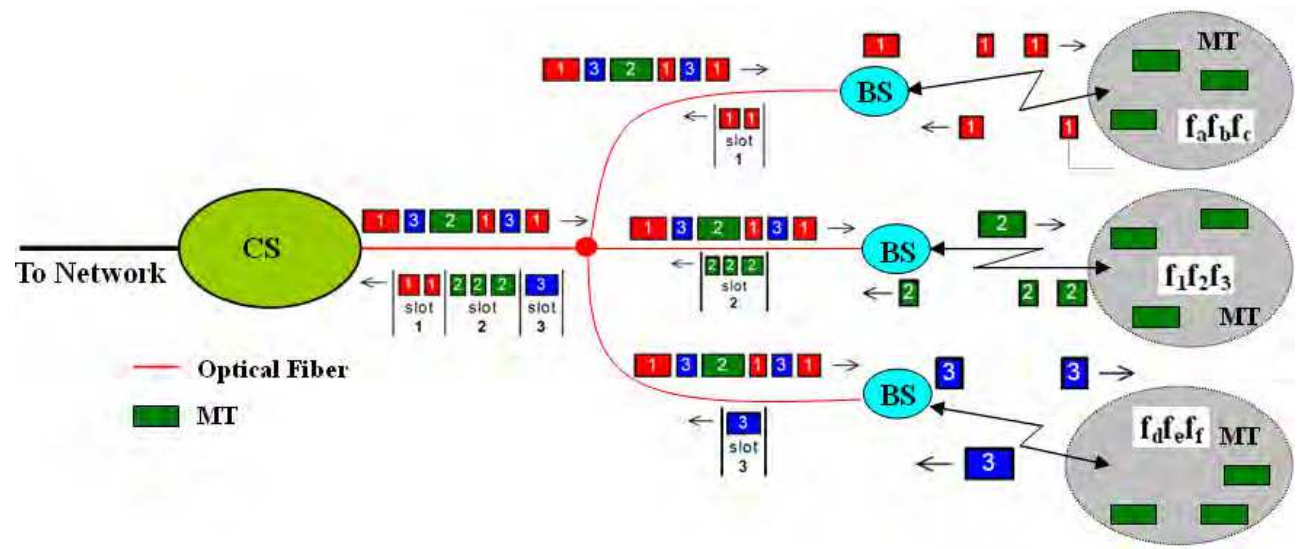

Fig. 18. Principle of bidirectional RoF system with distributed BSs based on TDM.

The basic priciple of TDM RoF system is like that of EPON, which is shown in Figure 19. But the realization of TDM in EPON is based on special protocols and techniques and both Optical Line Terminal (OLT) and Optical Network Unit (ONU) need to own the ability of data processing (Kramer, 2006). Because BS in RoF system corresponds to ONU in EPON, if each BS is in charge of data processing instead of a transparent interface, the system can not be cost-effective.

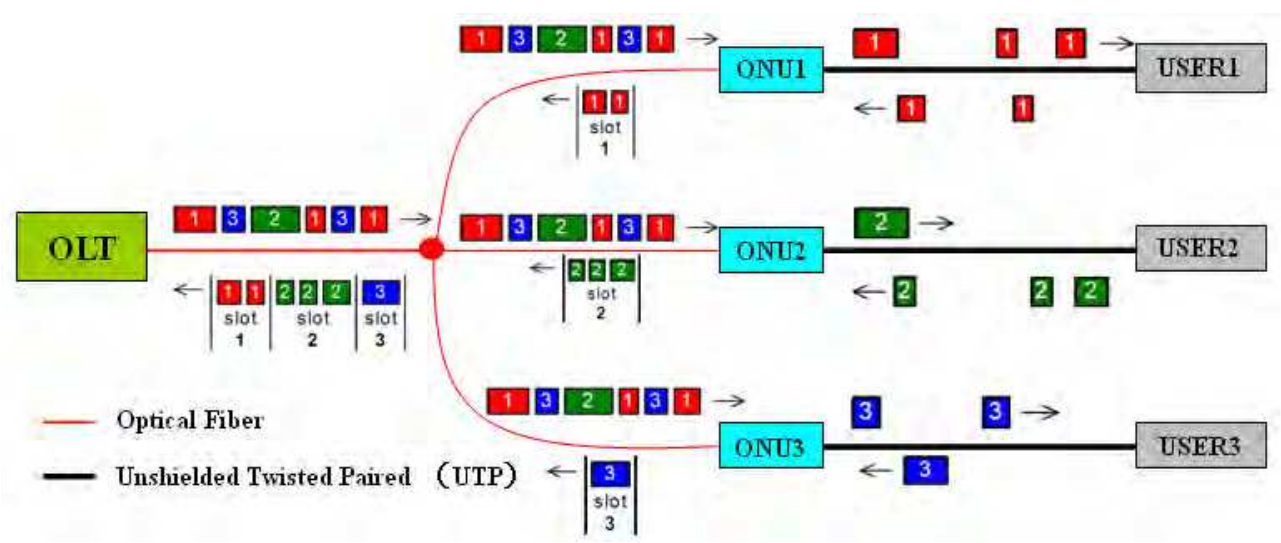

Fig. 19. The basic structure of EPON 
Many research works have been done to realize RoF system with distributed BSs incorporating Wavelength Division Multiplexing (WDM) (Stöhr et al., 1998; Griffin et al., 1999). Compared to incorporating TDM, a RoF system based on WDM does not need complex protocols to handle data and can make the structure of BS as simple as possible. The utilization of WDM can simplify the network architecture by using different wavelengths to feed different BSs, and greatly simplify network upgrade and maintenance by enabling the introduction of new services and the deployment of additional BSs (Nirmalathas et al., 2000). Figure 20 shows a star-tree architecture for a RoF system incorporating WDM. In the system, the fiber links from the CS form the star part of the architecture while the tree part is at the remote node (RN) with each branch feeding a different BS pico-cell. Each RN, as one arm of star feeds its group of BSs by its own unique WDM wavelengths for both the downlink and the uplink.

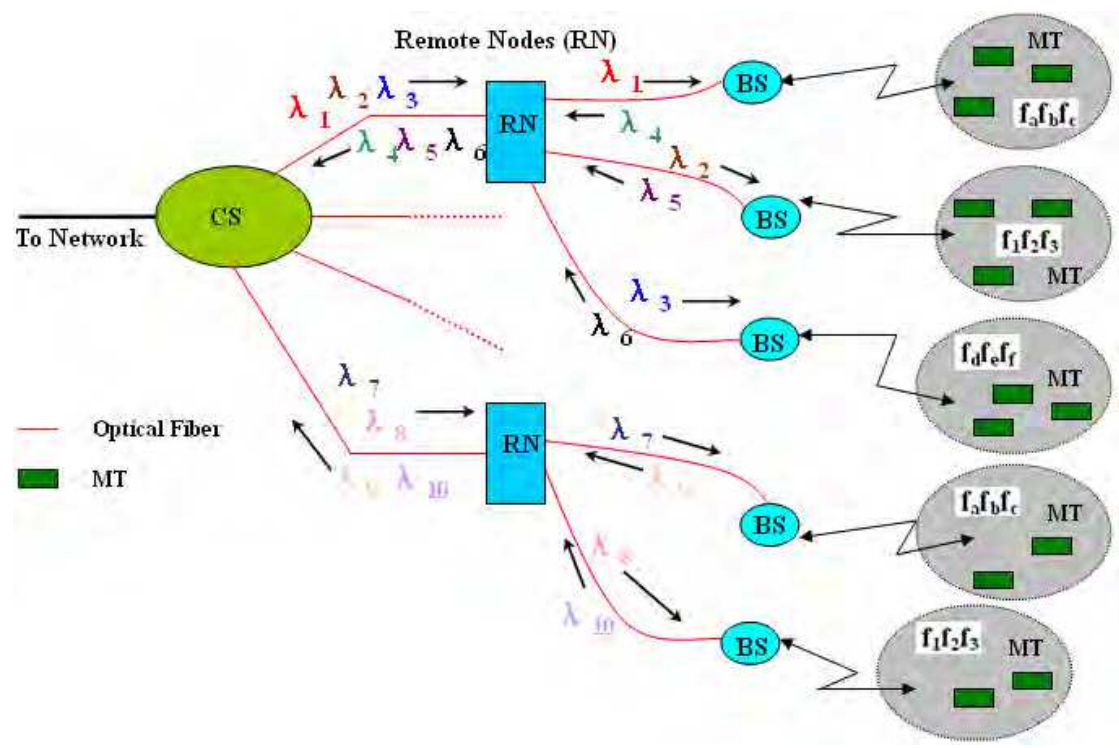

Fig. 20. Star-tree architecture for a RoF system incorporating WDM.

A WDM mm-wave RoF system with a star-tree architecture has been demonstrated (Smith et al., 1998). In this system, three SCM mm-wave signals each carrying 155-Mb/s data are transmitted in the RoF link. An alternative WDM RoF architecture is the ring network shown in Figure 21. The ring topology allows the allocation of a single wavelength to a particular BS and the wavelength routing is enabled via optical add-drop multiplexers (OADMs). The CS provides a number of wavelengths each carrying multiple modulated RF subcarriers. Uplink transmission is achieved by modulating uplink RF signals onto an optical carrier at the same BS wavelength, and adding it back into the ring via the OADM (Nirmalathas et al., 2000). 


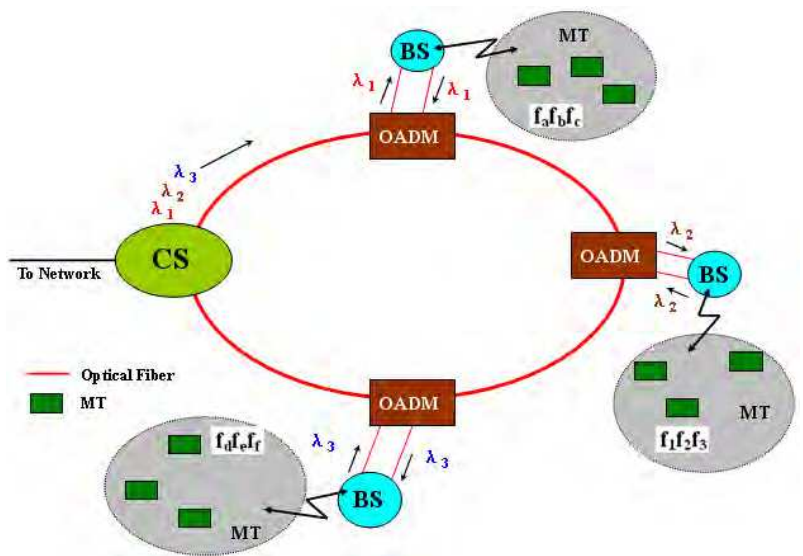

Fig. 21. Ring architecture for a RoF system incorporating WDM.

\subsubsection{Distributed mm-wave RoF system incorporating WDM and OFM}

OFM techniques have been demonstrated as the effiecient ways to yield mm-wave signals, as discussed in section 2.3. In this section, a distributed $40-\mathrm{GHz}$ RoF system and its MT design will be proposed in Figure 22 (The appropriate RF amplifiers are not drawn for simplicity). In this system, a high power 5-GHz RF signal is applied to DD-MZM. The bandwidth capacity of the system can achieve 2.5-GHz. CS broadcasts the downlink optical signals to each BS at one wavelength, which reduces the number of modulators such as DDMZM and IM in CS. BS as a transparent interface is only in charge of optical-electronic conversion work. In MT, Data Processing Unit demodulated the $2.5-\mathrm{GHz}$ signal and extracted the data frames sent with its ID number (MAC address).

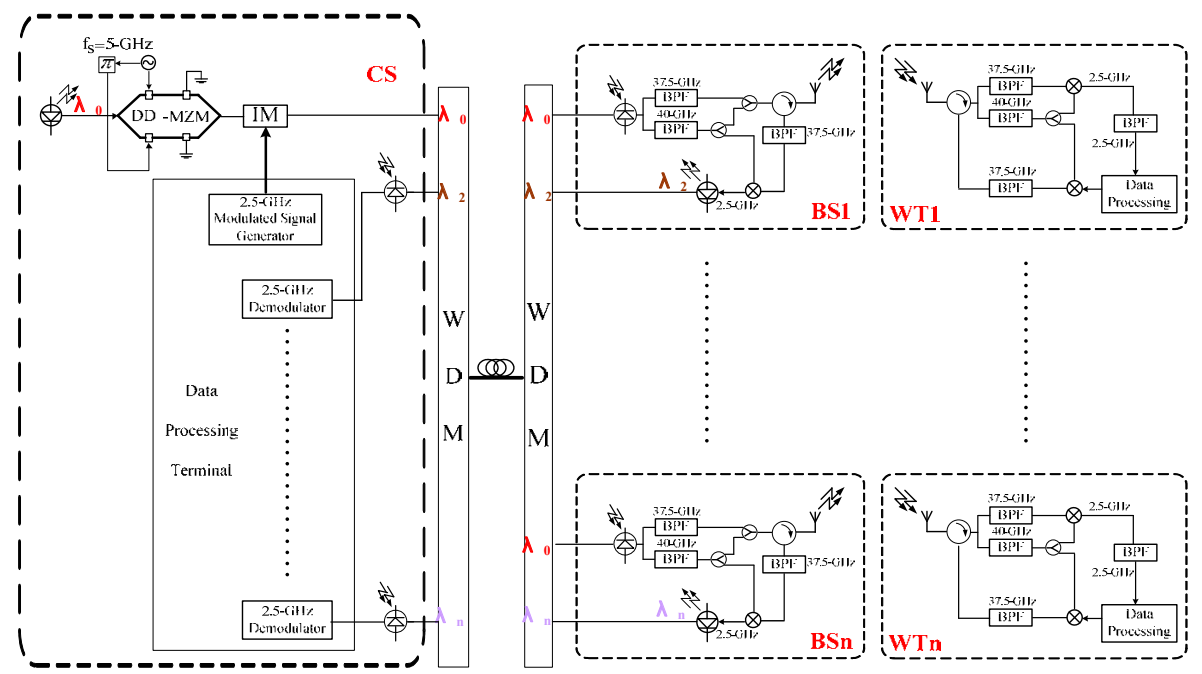

Fig. 22. Architecture of distributed 40-GHZ RoF system and the design of MT. 
Because the modulation scheme discussed in section 3.2 is adopted, pure 40-GHz reference can be yielded together with the 37.5-GHz modulated signal in BS. Unlike the BS design in section 3.1, both $40-\mathrm{GHz}$ carrier and $37.5-\mathrm{GHz}$ modulated signals are transmitted from BS to MT in this system. Therefore, the $40-\mathrm{GHz}$ carrier can be used as mm-wave reference for both BS and MT. In the uplink, each BS transmits the down-converted 2.5-GHz signal back to CS with a different wavelength.

\section{Millimeter-wave fading induced by fiber chromatic dispersion in RoF system}

The fiber chromatic dispersion is always one of critical problems in optical communications. Optical components at different frequencies travel through the fiber at different velocities. A pulse of light broadens and becomes distorted after passing through a single-mode fiber (Meslener, 1984). To mm-wave RoF system, the fiber chromatic dispersion causes the remarkable mm-wave fading (Schmuck, 1995).

\subsection{Analysis of chromatic dispersion in intensity modulated RoF system}

The intersity modulation schemes of yielding mm-wave signal have been introduced in Section 2.1. Those schemes may be sensitive to fiber chromatic dispersion. For example, an external optical modulator (MZM) is used to modulate CW optical signal with a RF signal. The electric field at the output of optical modulator is express as (Schmuck, 1995)

$$
E(t)=E_{c} \cos \left[d \cdot \frac{\pi}{2}+m \cdot \frac{\pi}{2} \cos \omega_{m} t\right] \cdot \cos \omega_{c} t
$$

where $E_{c}$ is the amplitude of electric field; $\omega_{c}$ is the central angular frequency of optical source; $\omega_{s}$ is the angular frequency of RF signal; $m=V_{m} / V_{\pi}$ is normalized amplitude of the driving RF signal; $d=V_{b} / V_{\pi}$ is the normalized bias voltage of the modulator; $V_{\pi}$ is the $\pi$ shift voltage of the modulator.

The electric field for $V_{b}=V_{\pi} / 2$, after the transmission over a fiber link can be expressed by Bessel functions

$$
E(t)=\frac{E_{c}}{\sqrt{2}} J_{0}(\beta) \cos \left(\omega_{c} t+\phi_{0}\right)-\frac{E_{c}}{\sqrt{2}} J_{1}(\beta)\left\{\cos \left[\left(\omega_{c}-\omega_{m}\right) t+\phi_{1}\right]+\cos \left[\left(\omega_{c}+\omega_{m}\right) t+\phi_{2}\right]\right\}
$$

where $\beta=m \pi / 2 ; \phi_{0}, \phi_{1}$ and $\phi_{2}$ represent the different phase delays of the optical components due to the fiber chromatic dispersion.

After photo-detection at the PD, the power of wished mm-wave signal can be approximately expressed as

$$
p \propto \cos ^{2}\left[\pi c D\left(\frac{f_{m}}{f_{c}}\right)^{2} z\right]=\cos ^{2}\left[\frac{\pi D \lambda_{c}^{2} f_{m}^{2} z}{c}\right]
$$


where $D$ represents the fiber group velocity dispersion parameter; $c$ is the velocity of light in vacuum; $\lambda_{c}$ is wavelength and $z$ is the fiber length. If parameters are chosen as: $c=3 \times 10^{8}-\mathrm{m} / \mathrm{s}$, $D=17-\mathrm{ps} /(\mathrm{km} \times \mathrm{nm}), \lambda_{c}=1550-\mathrm{nm}, f_{m}=40-\mathrm{GHz}$, the relation between the amplitude of mmwave and the transmission distance in fiber is shown in Figure 16. It shows that the amplitude of mm-wave changes with the transmission distance so fast that this mm-wave generation scheme can not be used in practice.

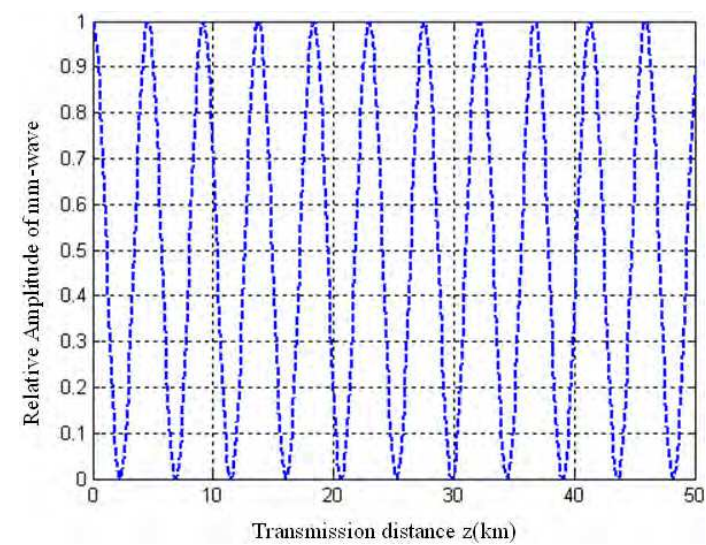

Fig. 16. The relative amplitude of $40-\mathrm{GHz} \mathrm{mm}$-wave varies with the fiber length

Many methods have been proposed to overcome the mm-wave signal fading induced by fiber chromatic dispersion. Smith et al. (1997) proposed a method to generate an optical carrier with single sideband (SSB) modulation by using a DD-MZM, biased at quadrature point, and applied with RF signals, $\pi / 2$ out of phase to its two electrodes. The RF power degradation due to fiber dispersion was observed to be only $15-\mathrm{dB}$ when using the technique to send 2 to $20-\mathrm{GHz}$ signals over $79.6-\mathrm{km}$ of fiber. By using an optical filter to depress one sideband. SSB optical modulation is realized and demonstrated by Park et al. (1997). Moreover, stimulated Brillouin scattering (SBS), a nonlinear phenomenon in optical fiber was applied to realize SSB modulation by Yonenaga \& Takachio (1993).

\subsection{Fiber chromatic dispersion in OFM techniques}

In this section, the chromatic dispersion in OFM techinques will be discussed. According to the basic arrangement of optical frequency sweeping technique, shown in Figure 6, the equation (2) can also be expressed as (Walker et al., 1992)

$$
E_{\text {in }}(t)=f\left(\omega_{s} t\right) \exp \left(j \omega_{c} t\right)=\sum_{n=-\infty}^{+\infty} F_{n} \exp \left[j\left(\omega_{c}+n \omega_{s}\right) t\right]
$$

where the harmonic components $F_{n}$ is given by:

$$
\begin{gathered}
F_{n}=\frac{1}{2 \pi} \int_{-\pi}^{\pi} f(\theta) \exp (-j n \theta) d \theta \\
f(\theta)=E_{c} \exp (j \beta \cos \theta)+E_{c} \exp \left[j \beta \cos \left(\theta-\omega_{s} \tau\right)-j \omega_{c} \tau\right]
\end{gathered}
$$


The fiber transfer characteristic can be written in the form

$$
H(\omega)=\exp \left[-j\left(k_{0}+k_{1}\left(\omega-\omega_{c}\right)+\frac{k_{2}}{2}\left(\omega-\omega_{c}\right)^{2}+\cdots\right) z\right]
$$

where the first term is a constant phase shift, the second term is constant propagation delay and the third term is the first order dispersion of optical fiber. At the angular frequencies of side modes in the light-wave, $H(\omega)$ has the values:

$$
H_{n}=H\left(\omega_{c}+n \omega_{s}\right)=\exp \left[-j\left(k_{0}+k_{1} n \omega_{s}+\frac{k_{2}}{2} n^{2} \omega_{s}^{2}\right) z\right]=\exp \left[-j\left(k_{0} z+k_{1} n \omega_{s} z+n^{2} \varphi\right]\right.
$$

where $\varphi=k_{2} \omega_{s}^{2} z / 2$ represents the fiber dispersion at the angular frequency of the first sidemode.

The first order dispersion constant $D$ of fiber is related to $k_{2}$ by the expression $D=-2 \pi c k_{2} / \lambda_{c}^{2}$, therefore $\varphi$ is related to $D$ by

$$
\varphi=-\frac{\omega_{s}^{2} D \lambda_{c}^{2}}{4 \pi c} z
$$

where $c$ is the light velocity in vacuum, $z$ is the transmission distance in fiber and $\lambda_{c}$ is the working wavelength.

The electric field of light-wave at output of the fiber is

$$
E_{\text {out }}(t)=\sum_{n=-\infty}^{\infty} F_{n} H_{n} \exp \left[j\left(\omega_{c}+n \omega_{s}\right) t\right]
$$

The photo-current produced in PD is

$$
i_{d}(t) \propto E_{\text {out }}(t) E_{\text {out }}^{*}(t)=\sum_{n=-\infty}^{\infty} \sum_{m=-\infty}^{\infty} F_{n} F_{m}^{*} H_{n} H_{m}^{*} \exp \left[j(n-m) \omega_{s} t\right]
$$

Setting $p=n-m$ and substituting (20) and (23) for $F_{n}$ and $H_{n}$ in (26) gives

$$
\begin{aligned}
i_{d}(t) & \propto \sum_{p=-\infty}^{\infty} \frac{1}{2 \pi} \int_{-\pi}^{\pi} f(\theta-p \varphi) f(\theta+p \varphi)^{*} \exp (-j p \theta) d_{\theta} \exp \left(j p \omega_{s}\left(t-k_{1} z\right)\right) \\
& =\sum_{p=-\infty}^{\infty} I_{p} \exp \left(j p \omega_{s}\left(t-k_{1} z\right)\right)
\end{aligned}
$$

Hence the amplitude of $p$-th harmonic in photo-current after transmission over the fiber becomes

$$
I_{p}=\frac{1}{2 \pi} \int_{-\pi}^{\pi} f(\theta-p \varphi) f(\theta+p \varphi)^{*} \exp (-j p \theta) d \theta
$$

Substituting (21) for $f(\theta)$ in (28) and performing the integration give 


$$
\begin{aligned}
I_{p}= & E_{c}^{2}\left\{J_{p}(2 \beta \sin p \varphi)+\exp \left(-j p \omega_{s} \tau\right) J_{p}(2 \beta \sin p \varphi)\right. \\
& +\exp \left(j \omega_{c} \tau-j p \frac{\omega_{s} \tau}{2}\right) J_{p}\left(2 \beta \sin \left(p \varphi-\frac{\omega_{s} \tau}{2}\right)\right) \\
& \left.+\exp \left(-j \omega_{c} \tau-j p \frac{\omega_{s} \tau}{2}\right) J_{p}\left(2 \beta \sin \left(p \varphi+\frac{\omega_{s} \tau}{2}\right)\right)\right\}
\end{aligned}
$$

So the pth harmonic can be approximately expressed by

$$
F_{p}=I_{p} \exp \left(j p \omega_{s} t\right)+I_{-p} \exp \left(-j p \omega_{s} t\right)
$$

Applying the parity of Bessel function to equation (38), $F_{n}$ can be written as

$$
\begin{aligned}
F_{p}= & 2 E_{c}^{2}\left\{J_{p}(2 \beta \sin p \varphi)\left[\cos p \omega_{s} t+\cos \left(p \omega_{s} t-p \omega_{s} \tau\right)\right]\right. \\
& +J_{p}\left(2 \beta \sin \left(p \varphi+\frac{\omega_{s} \tau}{2}\right)\right) \cos \left(p \omega_{s} t-p \frac{\omega_{s} \tau}{2}-\omega_{c} \tau\right) \\
& \left.+J_{p}\left(2 \beta \sin \left(p \varphi-\frac{\omega_{s} \tau}{2}\right)\right) \cos \left(p \omega_{s} t-p \frac{\omega_{s} \tau}{2}+\omega_{c} \tau\right)\right\}
\end{aligned}
$$

The intensity modulation depth $M_{p}$ is defined as $M_{p}=\left|F_{p} / F_{0}\right|$. In the condition that the optimized condition $\left(\omega_{c} \tau=k \pi, \omega_{s} \tau=\pi\right)$ for optical frequency sweeping technique is satisfied, the intensity

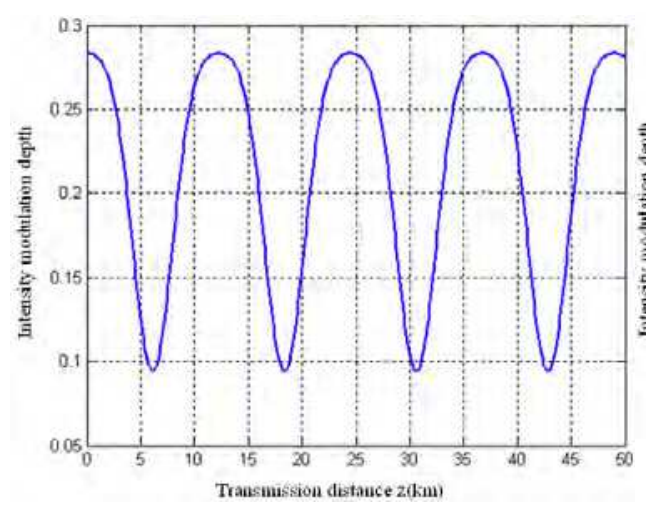

(a)

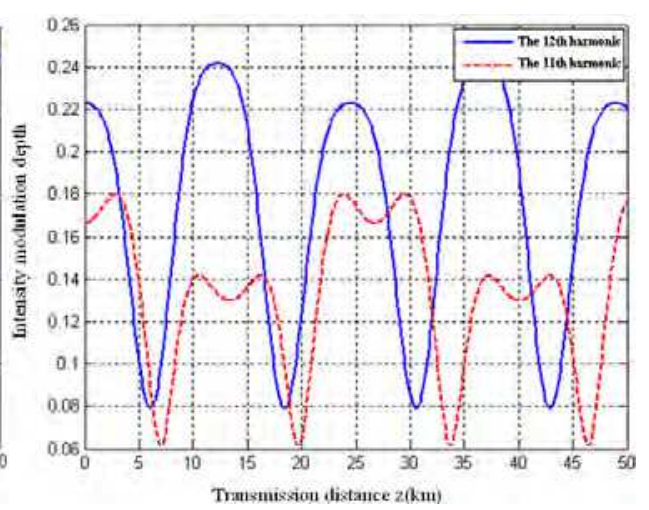

(b)

Fig. 17. The intensity modulation depth of 12th harmonic in the (a) satisfied condition, (b) unsatisfied condition.

modulation depth of 12th harmonic with transmission distance is shown in Figure 17 (a). Figure (b) shows the intensity modulation depth in the unsatisfied condition and the odd harmonics appear.

Lin et al. (2008) analyzed the mm-wave fading caused by fiber chromatic dispersion in the OFM scheme using nonlinear modulation of DD-MZM. The result is drawn in Figure 18, 
together with the result of double side-modes IM (without carrier depression) for comparison. It can be seen in Figure 18 that in the double side-modes IM scheme the amplitude of generated $40-\mathrm{GHz}$ mm-wave behaves $100 \%$ fading with periodic zeros at different fiber lengths. In contrast, in OFM scheme using DD-MZM, the amplitude fading of generated $40-\mathrm{GHz}$ mm-wave is much weaker, only $30 \%$ and without zeros. Furthermore, the minimum amplitude happens in much longer period. This means that OFM by using DDMZM is a good mm-wave generation method with tolerability to fiber chromatic dispersion. Conceptually, OFM by using DD-MZM is such a system that generation of mm-wave is the superposition of several mm-waves generated by self-heterodyne of several pairs of optical side-modes. So the interference of several mm-waves at the same frequency results in only a little amplitude fading.

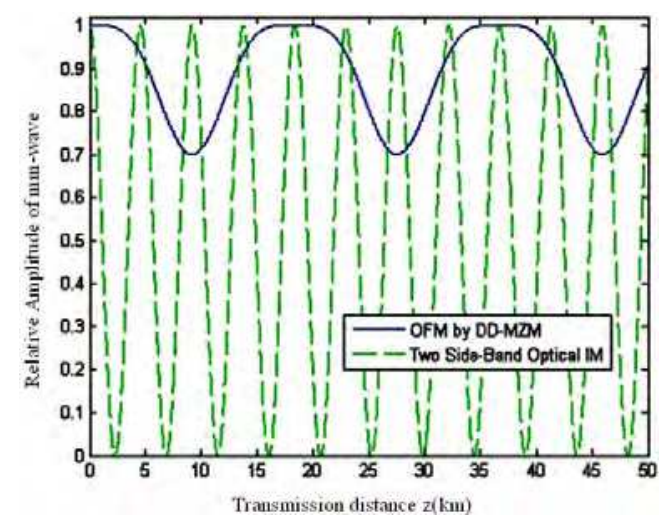

Fig. 18. Amplitude of $40 \mathrm{GHz} \mathrm{mm}$-wave varies with fiber length in double side-modes IM scheme and DD-MZM OFM scheme.

\section{Fast handover in mm-wave RoF system}

There is much more free space loss at mm-wave band than that at 2.4-GHz or 5-GHz, since free space loss increases drastically with frequency. In principle this higher free space loss can be compensated for by the use of antennas with stronger pattern directivity while maintaining small antenna dimensions. When such antennas are used, however, antenna obstruction (e.g., by a human body) and mispointing may easily cause a substantial drop of received power, which may nullify the gain provided by the antennas. This effect is typical for $\mathrm{mm}$-wave signals because the diffraction of $\mathrm{mm}$-wave signals (i.e., the ability to bend around edges of obstacles) is weak (Smulders, 2002), so a mm-wave communication network has many characteristics quite different from conventional wireless LANs (WLANs) operating in 2.4 or $5-\mathrm{GHz}$ bands.

Due to the free space loss of mm-wave signal, the coverage of BS, as pico-cell has been smaller than that of Access Point (AP) in current WLAN. The small size of pico-cell induces the large number of BSs and frequent handovers of MT from one pico-cell to another. As a result, the key point in designing the Medium Access Control (MAC) protocol for mm-wave RoF system is to provide efficient and fast handover support. A MAC protocol based on Frequency Switching (FS) codes can realize fast handover and adjacent pico-cells employ 
orthogonal FS codes to avoid possible co-channel interference (Kim \& Wolisz, 2003). A moveable cells scheme based on optical switching architecture can realize the handover in the order of ns or $\mu$ s (Lannoo et al., 2004), which is suitable to all MTs moving at the same speed, for example in a train scenario. In this way, MT can operate on the same frequency during the whole connection and avoid the fast handovers. Based on moveable cells scheme, Yang \& Liu (2008) proposed a further scheme, in which the adjacent pico-cells are grouped as a larger cell, and along the railway all the BS in this larger cell use the same frequency channel. When $n$ adjacent pico-cells are grouped, times of handover can be decreased $n$-fold.

\section{Conclusion}

In this chapter, many technical issues about the mm-wave RoF systems are presented. Firstly, three kinds of mm-wave generation techniques are introduced. In those techniques, OFM techniques realized by optical frequency sweeping and nonlinear modulation of DD-MZM are mainly discussed and the latter is proved to be a more stable and cost-efficient way to yield signal at the mm-wave band. Unlike most research works by now only concentrating on the downlink of RoF system, the design of several bidirectional mm-wave RoF systems is described which deals with the uplink as optical transport of IF signal, generated by down-conversion of $\mathrm{mm}$-wave signal. The information-bearing $\mathrm{mm}$-wave for radiation and the reference mm-wave for down-conversion are all generated in BS by OFM. Then, two multiplexing techniques, WDM and SCM are introduced to mm-wave RoF systems. Star-tree and ring architectures are adopted in mm-wave RoF systems to realize the distributed BSs. After showing the large bandwidth capacity at mm-wave band provided by OFM techniques, incorporating SCM to RoF system is demonstrated to improve the utilization ratio of large bandwidth. Considering the influence of chromatic dispersion in fiber on mm-wave fading, a common analysis on the effect of fiber chromatic dispersion to mm-wave generation techniques (i.e., intensity modulation and OFM) are given and OFM by using DD-MZM is proved to be tolerable to fiber chromatic dispersion. Due to the great free space loss of signal at mm-wave band, the coverage of each BS is very small and the handover of MT becomes a problem. To meet the real-time communication requirements for mm-wave systems, several MAC protocols suitable either to efficient and fast handover or to moveable cells schemes, which make the MT avoid the fast handover problem, are introduced.

\section{Acknowledgements}

This work was surpported by the National Natural Science Foundation of China (60377024 and 60877053), and Shanghai Leading Academic Discipline Project (08DZ1500115).

\section{References}

Braun, R.-P.; Grosskopf, G.; Heidrich, H.; von Helmolt, C.; Kaiser, R.; Kruger, K.; Kruger, U.; Rohde, D.; Schmidt, F.; Stenzel, R. \& Trommer, D. (1998). Optical microwave generation and transmission experiments in the 12- and $60-\mathrm{GHz}$ region for wireless communications, Microwave Theory and Techniques, IEEE Transactions on, Vol. 46, No. 4, pp. 320-330. 
Doi, M.; Hashimoto, N. ; Hasegawa, T. ; Tanaka, T. \& Tanaka, K (2007). 40 Gb/s low-drivevoltage LiNbO3 optical modulator for DQPSK modulation format. in Optical Fiber Communication Conference and Exposition and The National Fiber Optic Engineers Conference, OSA Technical Digest Series (CD), paper OWH4.

Elrefaie, A.F.; Wagner, R.E.; Atlas, D.A. \& Daut, D.G. (1988). Chromatic dispersion limitations in coherent lightwave transmission systems, Lightwave Technology, Journal of, Vol. 6, No. 5, pp. 704-709, 1988.

Fuster, J.M.; Marti, J.; Candelas, P.; Martinez, F.J. \& Sempere, L. (2001). Optical generation of electrical modulation formats, 27th European Conference on Optical Communication (ECOC 2001), pp. 536-537, 2001.

Garcia Larrode, M.; Koonen, A.M.J.; Vegas Olmos, J.J.; Tafur Monroy, I. \& Schenk, T.C.W. (2005). RF bandwidth capacity and SCM in a radio-over-fibre link employing optical frequency multiplication, Conference on Optical Communication, 2005 (ECOC 2005), Vol. 3, pp. 681-682, Sep. 25-29, 2005.

Gliese, U.; Nielsen, T. N.; Bruun, M.; Lintz Christensen, E.; Stubkjaer, K. E.; Lindgren, S. \& Broberg, B. (1992). A wideband heterodyne optical phase-locked loop for generation of 3-18 GHz microwave carriers, IEEE Photonics Technology Letters, Vol. 4, No. 8, pp. 936-938.

Gliese, U.; Norskov, S. \& Nielsen, T.N. (1996). Chromatic dispersion in fiber-optic microwave and millimeter-wave links, Microwave Theory and Techniques, IEEE Transactions on, Vol. 44, No. 10, pp. 1716-1724.

Griffin, R.A.; Lane, P.M. \& O’Reilly, J.J. (1999). Radio-over-fiber distribution using an optical millimeterwave/DWDM overlay, OFC 1999, Paper WD6-1, 1999.

Hartmannor, P. ; Webster, M. ; Wonfor, A. ; Ingham, J.D. ; Penty, R.V. ; White, I.H. ; Wake, D. \& Seeds, A.J. (2003). Low cost multimode fibre based wireless LAN distribution system using uncooled, directly modulated DFB laser diodes, 2003 European Conference on Optical Communication (ECOC 2003), Sep. 21-25, 2003.

Juha Rapeli (2001). Future directions for mobile communications business, technology and research, Wireless Personal Communications, Vol. 17, No. 2-3, pp.155-173.

Kim, H.B. \& Wolisz, A., Performance evaluation of a MAC protocol for radio over fiber wireless LAN operating in the $60-\mathrm{GHz}$ band, Global Telecommunications Conference, 2003 (GLOBECOM '03), Vol. 5, pp. 2659-2663, Dec. 1-5, 2003.

Kitayama, K. (1998). Architectural considerations of radio-on-fiber millimeter-wave wireless access systems, International Symposium on Signals, Systems, and Electronics, 1998 (ISSSE 98), pp.

Kramer, G. (2006). What is next for Ethernet PON?, The Joint Intenational Conference on Optical Internet and Next Generation Network, 2006 (COIN-NGNCON 2006), pp. 49-54, Jul. 913, 2006.

Kuri, T.; Kitayama, K.; Stohr, A. \& Ogawa, Y. (1999). Fiber-optic millimeter-wave downlink system using $60 \mathrm{GHz}$-band external modulation, Lightwave Technology, Journal of, Vol. 17, No. 5, pp. 799-806.

Lannoo, B.; Colle, D.; Pickavet, M. \& Demeester, P. (2004). Optical switching architecture to realize "moveable cells" in a radio-over-fiber network, 6 th International Conference on Transparent Optical Networks, 2004, Vol. 2, pp. 2-7, Jul. 4-8, 2004. 
Larrode, M.G.; Koonen, A.M.J.; Olmos, J.J.V.; Verdurmen, E.J.M. \& Turkiewicz, J.P. (2006). Dispersion tolerant radio-over-fibre transmission of 16 and 64 QAM radio signals at $40 \mathrm{GHz}$, Electronics Letters, Vol. 42, No. 15, pp. 872-874.

Lin, Ru-jian; Zhu, Mei-wei; Zhou, Zhe-yun \& Ye, Jia-jun (2008). Theoretic and experimental study on mm-wave radio over fiber system based on OFM, Proc. SPIE, Vol. 7137, 71371M (2008), DOI:10.1117/12.807835.

Meslener, G. (1984). Chromatic dispersion induced distortion of modulated monochromatic light employing direct detection, Quantum Electronics, Journal of, Vol. 20, No. 10, pp. 1208-1216, 1984.

Nirmalathas, A.; Lim, C.; Novak, D.; Castleford, D.; Waterhouse, R. \& Smith, G. (2000). Millimeter-wave fiber-wireless access systems incorporating wavelength division multiplexing, Microwave Conference, 2000 Asia-Pacific, pp. 625-629, 2000.

Ogusu, M.; Inagaki, K.; Mizuguchi, Y. \& Ohira, T. (2003). Carrier generation and data transmission on millimeter-wave bands using two-mode locked Fabry-Perot slave lasers, IEEE transactions on microwave theory and techniques, Vol. 51 (1), No. 2, pp. 382-391.

Olshansky, R.; Lanzisera, V.A. \& Hill, P.M. (1989). Subcarrier multiplexed lightwave systems for broad-band distribution, Lightwave Technology, Journal of , Vol. 7, No. 9, pp. 1329-1342, 1989.

O'Rcilly, J.J.; Lane, P.M.; Heidemann, R. \& Hofstetter, R. (1992). Optical generation of very narrow linewidth millimetre wave signals, Electronics Letters, Vol. 28, No. 25, pp. 2309-2311.

Park, J.; Sorin, W.V.\& Lau, K.Y. (1997). Ellimination of the fiber chromatic dispersion penalty on 1550nm millimeter-wave optical transmission, Electronics Letters, Vol. 33, No. 6, pp. 512-513, 1997.

Schmuck, H. (1995). Comparison of optical millimeter-wave system concepts with regard to chromatic dispersion, Eletronics Letters, Vol. 31, No. 21, pp.1848-1849, 1995.

Smith, G.H.; Novak, D. \& Ahmed, Z. (1997). Techniques for optical SSB generation to overcome dispersion penalties in fibre-radio systems, Electronics Letters, Vol. 33, No. 1, pp. 74-75, 1997.

Smith, G.H.; Novak, D. \& Lim, C., A (1998). Millimeter-wave full-duplex fiber-radio star-tree architecture incorporating WDM and SCM, Photonics Technology Letters, IEEE, Vol. 10, No. 11, pp. 1650-1652, 1998.

Smulders, P. (2002). Exploiting the $60 \mathrm{GHz}$ band for local wireless multimedia access: prospects and future directions, Communications Magazine, IEEE, Vol. 40, No. 1, pp.140-147.

Sun, C.K.; Orazi, R.J. \& Pappert, S.A. (1996). Efficient microwave frequency conversion using photonic link signal mixing, IEEE Photonics Technology Letters, Vol. 8, No. 1, pp. 154-156.

Stöhr, A.; Kitayama, K. \& Jäger, D. (1998). Error-free full-duplex optical WDM-FDM transmission using an EA-transceiver, International Topical Meeting on Microwave Photonics, 1998 (MWP 98), pp. 37-40, Oct. 12-14, 1998.

Ton Koonen; Anthony Ng"oma; Peter Smulders; Henrie van den Boom; Idelfonso Tafur Monroy \& Giok-Djan Khoe (2003). In-house networks using multimode Polymer Optical Fibre for broadband wireless services, Photonic Network Communications, Vol. 5, No. 2, pp. 177-187. 
Tsuzuki, K.; Sano,K.; Kikuchi, N.; Kashio, N.; Yamada, E.; Shibata, Y.; Ishibashi, T.; Tokumitsu, M. \& Yasaka, H. (2006). 0.3 Vpp single-drive push-pull InP MachZehnder modulator module for 43-Gbit/s systems, Optical Fiber Communication Conference and Exposition and The National Fiber Optic Engineers Conference, Technical Digest (CD) (Optical Society of America, 2006), paper OWC2.

Walker, N.G.; Wake, D. \& Smith, I. C. (1992). Efficient millimeter-wave signal generation through FM-IM conversion in dispersive optical fiber links, Electronics Letters, Vol. 28, No. 21, pp. 2027-2028, 1992.

Williams, K.J.; Goldberg, L.; Esman, R.D.; Dagenais, M. \& Weller, J.F. (1989). 6-34 GHz offset phase-locking of Nd:YAG $1319 \mathrm{~nm}$ nonplanar ring lasers, Electronics Letters, Vol. 25, No. 18, pp. 1242-1243.

Xiu, Ming-lei \& Lin, Ru-jian (2007). Report on 40GHz-RoF bidirectional transmission experiment system with pilot tone, Conference on Lasers and Electro-Optics - Pacific Rim, 2007. CLEO/Pacific Rim 2007, pp.1-2, Aug. 26-31, 2007.

Yang, Chunyong \& Liu, Deming (2008). Cell extension for fast moving users in a RoF network, Proc. SPIE 7278, 72781E (2008), DOI:10.1117/12.823294.

Yonenaga, K. \& Takachio, N. (1993). A fiber chromatic dispersion compensation technique with an optical SSB transmission in optical homodyne detection systems, Photonics Technology Letters, Vol. 5, No. 8, pp. 949-951, 1993.

Yungsoo Kim; Byung Jang Jeong; Jaehak Chung; Chan-Soo Hwang; Ryu, J.S.; Ki-Ho Kim; Young Kyun Kim (2003). Beyond 3G: vision, requirements, and enabling technologies, Communications Magazine, IEEE , Vol. 41, No. 3, pp. 120-124.

Zhou, Zheyun ; Lin, Rujian \& Ye Jiajun (2008). A novel optical QPSK modulation scheme for millimeter-wave Radio-Over-Fiber system, 2008 Asia Optical Fiber Communication and Optoelectronic Exposition and Conference, OSA Technical Digest (CD), paper SaK12.

Zhu, Mei-wei; Lin, Ru-jian; Ye, Jia-jun \& Xiu, Minglei (2008). Novel millimeter-wave radioover-fiber system using dual-electrode Mach-Zehnder modulator for millimeterwave generation. Opto-Electronic Engineering, Vol. 35, No. 4, pp. 126-130. 


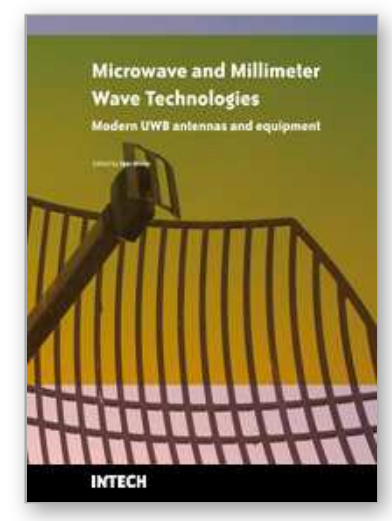

\section{Microwave and Millimeter Wave Technologies Modern UWB antennas and equipment}

Edited by Igor Mini

ISBN 978-953-7619-67-1

Hard cover, 488 pages

Publisher InTech

Published online 01, March, 2010

Published in print edition March, 2010

\section{How to reference}

In order to correctly reference this scholarly work, feel free to copy and paste the following:

Haoshuo Chen, Rujian Lin and Jiajun Ye (2010). Millimeter-Wave Radio over Fiber System for Broadband Wireless Communication, Microwave and Millimeter Wave Technologies Modern UWB antennas and equipment, Igor Mini (Ed.), ISBN: 978-953-7619-67-1, InTech, Available from:

http://www.intechopen.com/books/microwave-and-millimeter-wave-technologies-modern-uwb-antennas-andequipment/millimeter-wave-radio-over-fiber-system-for-broadband-wireless-communication

\section{INTECH}

open science | open minds

\section{InTech Europe}

University Campus STeP Ri

Slavka Krautzeka 83/A

51000 Rijeka, Croatia

Phone: +385 (51) 770447

Fax: +385 (51) 686166

www.intechopen.com

\section{InTech China}

Unit 405, Office Block, Hotel Equatorial Shanghai

No.65, Yan An Road (West), Shanghai, 200040, China

中国上海市延安西路65号上海国际贵都大饭店办公楼 405 单元

Phone: +86-21-62489820

Fax: +86-21-62489821 
(C) 2010 The Author(s). Licensee IntechOpen. This chapter is distributed under the terms of the Creative Commons Attribution-NonCommercialShareAlike-3.0 License, which permits use, distribution and reproduction for non-commercial purposes, provided the original is properly cited and derivative works building on this content are distributed under the same license. 